\title{
Modelling piezoelectric excitation in waveguides using the semi-analytical finite element method
}

\author{
Michał K. Kalkowski, Emiliano Rustighi, Timothy P. Waters \\ Institute of Sound and Vibration Research, University of Southampton, Southampton SO17 \\ $1 B J$, United Kingdom
}

\begin{abstract}
In this paper we present a wave-based technique for modelling waveguides equipped with piezoelectric actuators in which there is no need for common simplifications regarding their dynamic behaviour, the interaction with the waveguide or the bonding conditions. The proposed approach is based on the semianalytical finite element (SAFE) method. We developed a new piezoelectric element and employed the analytical wave approach to model the distributed electrical excitation and scattering of the waves at discontinuities. The model was successfully verified numerically and validated against an experiment on a beam-like waveguide with emulated anechoic terminations.

Keywords: wave propagation, piezoelectric excitation, semi-analytical finite element method, wave approach, smart structures, guided waves
\end{abstract}

\section{Introduction}

Piezoelectric actuators and sensors play a key role in many modern engineering systems in various areas ranging from non-destructive testing, through active control, energy harvesting to power ultrasonics. The electromechanical coupling in the piezoelectric material enables an active interaction with the structure providing the means of excitation and sensing its dynamic response. The ability to describe the interaction between the piezo patch and the host structure accurately is an essential aspect of both the design and operational stages of piezo equipped dynamic systems.

Preprint submitted to Computers and Structures

May 19, 2016 
Over the past decades this problem has been widely studied bringing a considerable number of modelling approaches. A review of these can be found in a paper by Huang et al. [1]. Among research published to date, two general approaches to the described problem can be distinguished [2]. The first group considers the dynamics of the structure and piezo actuation separately assuming that they are fully decoupled - a set of equivalent forces is applied to the substrate model. The second group of models accounts for the dynamic interaction between the actuator and the structure and is based on the solution to a boundary problem.

The majority of models belonging to the first group originate from the work by Crawley and de Luis [3] who formulated the famous quasi-static assumption stating that the actuator behaves quasi-statically provided that it operates at frequencies far below its own resonances and is thin and light compared to the host structure. The model implies that the dynamics of the actuator are neglected and that for thin bonding layers the shear load transfer is confined to the ends of the actuator (the so-called pin-force assumption). For other bonding conditions a shear lag parameter that describes the effectiveness of the shear load is proposed [3]. Modelling of more complex structures was facilitated by employing the frequency-domain spectral element method (SEM) to describe coupled flexural-longitudinal-shear vibration of homogeneous [4-6] or composite [7] beams based on Timoshenko and Mindlin-Hermann theories.

Piezo actuators have become a standard for high-frequency wave-based nondestructive testing (NDT) [8]. The high frequency regime is associated with more complex through-thickness field variations, therefore the limitations of elementary approximate theories needed to be overcome. To achieve this, one might use a higher-order analytical theory, such as the Mindlin plate theory [9] or employ hybrid techniques, e.g. the coupling between the finite element method and normal modes expansion [10]. A paper by Raghavan and Cesnik [11] discusses a model based on an integral transform solution to the full 3-D elasticity equations with the external forcing approximated using the pin-force assumption. Alternatively, Lamb wave excitation with a piezoelectric wafer can 
be treated as a boundary problem. Giurgiutiu [12] presented a methodology incorporating full Lamb wave equations solved for external piezo-induced traction excitation. Based on this contribution, the influence of the bonding layer has been further investigated in e.g. [13, 14].

Most of the aforementioned models do not take into account the dynamics of the piezo layer and the mutual interaction between the patch and the structure. Some studies attempting to include these phenomena have been presented in $[15,16]$ where wave propagation induced by piezoelectric elements attached to an elastic half-space was studied. An integral equation based methodology for modelling piezo-structure interaction that accounts for the dynamic contribution of the piezo actuator and can be applied both to a plate and an elastic half-space has been published by Glushkov et al. [17].

Apart from analytical solutions, piezoelectric excitation can well be modelled using a conventional finite element method (FE). Although this technique is versatile and allows for modelling complicated structures, it has important drawbacks in high frequency applications such as a very large computational cost and a limited insight into wave propagation phenomena. However, in other numerical methods, some of these can be circumvented, e.g. by using the timedomain spectral finite element method as proposed in [18-20] where the number of degrees of freedom required is significantly reduced. Recent advances in computational engineering resulted in an interesting work by Paćko et al. [21] where a hybrid local interaction simulation approach (LISA)-FE method has been implemented using parallel computing and graphical card processing offering significant time savings when compared to FE.

The wide range of models discussed above serve many purposes in SHM applications well, but have certain limitations. The motivation for developing the presented wave-based methodology originates from attempting engineering problems for which the modelling requirements are not entirely fulfilled by the available methods. These requirements include: (a) ability to model an arbitrarily shaped/multi-layered cross-section of the waveguide; (b) ability to model thick actuators operating close to resonance (i.e. include actuator's dynamics); 
(c) insight into the wave composition of the response; (d) straightforward implementation of infinite boundaries; (e) facilitating the assembly of piezo-actuated built-up waveguides.

The approach presented in this paper is based on the semi-analytical finite element (SAFE) method. We develop a piezoelectric SAFE element, that allows for modelling piezoelectric excitation directly in the wave domain and accounts for the dynamics of the actuator. The analytical wave approach $[22,23]$ is used to represent scattering of waves at the junctions and reflection at the boundaries. Consequently, the proposed framework enables modelling the dynamics of complex multi-component piezo-equipped structures in a wave-based manner. Alternatively, similar advantages can be gained from using the scaled boundary finite element method (SBFEM) which was employed e.g. for analysis of dynamic cracks in piezoelectric materials in [24] and for simulating guided waves interaction with defects [25].

SAFE is a well established method for mechanical waveguides. The plane of the cross-section is discretised using an FE-like procedure and the derived equations of motion are solved with the aid of the spatial Fourier transform (SFT) in the direction of propagation. Semi-analytical approaches have been used in various configurations in structural dynamics and acoustics, e.g. in the strip element method $[26,27]$ or infinite elements (see review in [28]). The SAFE method as used in this paper was proposed in the 1970s $[29,30]$ and was further developed and expanded in the following years by many researchers. More details of the method can be found in e.g. [31-34].

The proposed approach enables modelling the piezoelectric excitation in structures without the need for common assumptions on the dynamics of the actuator, the actuator/structure interaction and the bonding conditions. The coupled-field wave model originates from the piezoelectric constitutive equations, so that a distributed voltage can be applied directly. It also allows for modelling of the bonding conditions by an additional layer of either elastic/viscoelastic SAFE elements or spring SAFE elements [35]. Moreover, it is not limited to the plane strain assumption, which is often the case for the com- 
monly used models. The approximations in place result from the finite element discretisation of the cross-section, the quasi-electrostatic approximation [36] and the fact that the electric field in the propagation direction is neglected. Thanks to the wave-based formulations, the proposed model provides insight into the wave composition of the computed response and enables modelling wave propagation in multi-component waveguides. To the best knowledge of the authors such a methodology has not been proposed to date.

The paper is outlined as follows. Firstly, we present the derivation of the piezoelectric SAFE element in Sec. 2. The free wave solution and propagation characteristics are discussed in Sec. 3. In Sec. 4 we calculate the response to a distributed voltage excitation for an infinite elastic waveguide covered with a piezoelectric layer powered over a finite length. We introduce wave reflection and scattering in Sec. 5 in order to represent wave excitation with a finite actuator. The convergence of the proposed element is assessed in Sec. 6. In Sec. 7 we verify the developed technique against conventional finite element simulations and a model from the open literature. Finally, an illustrative model of an infinite beam-like waveguide with a finite piezoelectric actuator is validated with an experiment in Sec. 8. The wave-based nature of the model is exploited to analyse the experimental results in Sec. 9. We draw a set of conclusions summarising the contributions of the paper in Sec. 10.

\section{Semi-analytical finite element formulation for an elastic waveguide with piezoelectric coupling}

The mathematical framework for the SAFE method for an elastic waveguide with piezoelectric coupling is developed below. It is assumed that the crosssection of the structure lies in the $x-y$ plane and that the wave propagates along the $z$ direction (see Fig. 1). The cross-section in general can be zero-, oneor two-dimensional. Only the two dimensional case is presented in this work, however the other cases can easily be recovered by removing appropriate degrees of freedom and altering the element shape functions. 


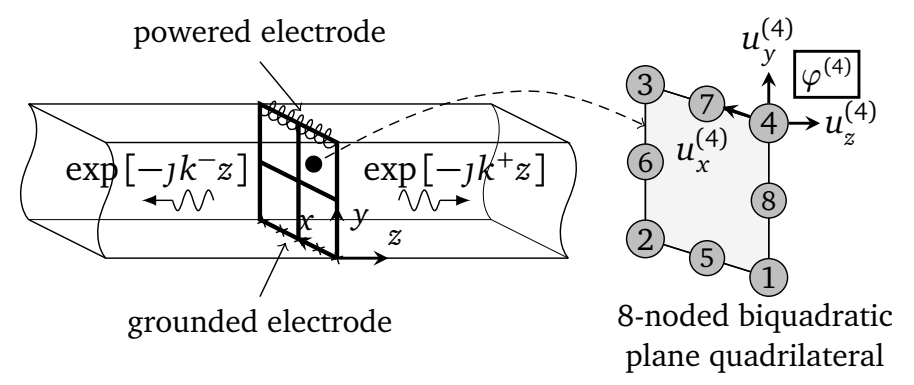

Figure 1: Piezoelectric SAFE element - the coordinates and degrees of freedom.

\subsection{Cross-section discretisation}

The displacement and strain fields in the waveguide are defined as [31]

$$
\begin{aligned}
\mathbf{u} & =\left[\begin{array}{lll}
u_{x} & u_{y} & u_{z}
\end{array}\right]^{\top} \\
\boldsymbol{\epsilon} & =\left[\begin{array}{llllll}
\epsilon_{x x} & \epsilon_{y y} & \epsilon_{z z} & \gamma_{y z} & \gamma_{x z} & \gamma_{x y}
\end{array}\right]^{\top}
\end{aligned}
$$

where the time-harmonic term $\mathrm{e}^{\jmath \omega t}$ is omitted for brevity.

The cross-section is discretised in an FE-like manner using elements and shape functions chosen according to the type of analysis. In this work an 8node quadrilateral biquadratic element was used for which the shape functions may be found in any standard textbook on the finite element method, e.g. [37]. Linear elements can also be used, however an accurate approximation of the wave field at high frequencies can be achieved at lower cost with quadratic elements.

The field variables from Eq. (1) are expressed using element shape functions (in the cross-section plane) and nodal degrees of freedom (DOFs) as implied by the FE approximation. Denoting the nodal displacements vector as $\mathbf{q}$, the displacement at any point within the SAFE element is

$$
\mathbf{u}(x, y, z)=\mathbf{N}(x, y) \mathbf{q}(z)
$$

where $\mathbf{N}(x, y)$ is the displacement shape function matrix.

Following from Eq. (2) one writes the strain in terms of the shape functions 
and the nodal DOFs as

$$
\begin{aligned}
\boldsymbol{\epsilon}(x, y, z)= & {\left[\mathbf{L}_{x} \frac{\partial}{\partial x}+\mathbf{L}_{y} \frac{\partial}{\partial y}+\mathbf{L}_{z} \frac{\partial}{\partial z}\right] \mathbf{u}(x, y, z)=} \\
& {\left[\mathbf{L}_{x} \mathbf{N}_{, x}+\mathbf{L}_{y} \mathbf{N}_{, y}+\mathbf{L}_{z} \frac{\partial}{\partial z} \mathbf{N}\right] \mathbf{q}(z)=} \\
& \mathbf{B}_{1} \mathbf{q}(z)+\mathbf{B}_{2} \frac{\partial}{\partial z} \mathbf{q}(z)
\end{aligned}
$$

where $\mathbf{N}_{, x}=\frac{\partial}{\partial x} \mathbf{N}$ and $\mathbf{L}_{x}, \mathbf{L}_{y}, \mathbf{L}_{z}$, are

$$
\mathbf{L}_{x}=\left[\begin{array}{ccc}
1 & 0 & 0 \\
0 & 0 & 0 \\
0 & 0 & 0 \\
0 & 0 & 0 \\
0 & 0 & 1 \\
0 & 1 & 0
\end{array}\right] \quad \mathbf{L}_{y}=\left[\begin{array}{ccc}
0 & 0 & 0 \\
0 & 1 & 0 \\
0 & 0 & 0 \\
0 & 0 & 1 \\
0 & 0 & 0 \\
1 & 0 & 0
\end{array}\right] \quad \mathbf{L}_{z}=\left[\begin{array}{lll}
0 & 0 & 0 \\
0 & 0 & 0 \\
0 & 0 & 1 \\
0 & 1 & 0 \\
1 & 0 & 0 \\
0 & 0 & 0
\end{array}\right]
$$

In a piezoelectric element each node has an additional degree of freedom representing the electrical potential $\varphi$. Therefore, the electrical potential in the waveguide $\vartheta$ is expressed using potential shape functions $\mathbf{N}_{\varphi}$ and nodal potential $\varphi$ as

$$
\boldsymbol{\vartheta}(x, y, z)=\mathbf{N}_{\varphi}(x, y) \varphi(z)
$$

\subsection{Constitutive equations}

The constitutive equations for the piezoelectric material are written in matricial ' $e$ ' form [38]

$$
\begin{aligned}
& \boldsymbol{\sigma}=\mathbf{C}^{\mathrm{E}} \boldsymbol{\epsilon}-\mathbf{e}^{\top} \mathbf{E} \\
& \mathbf{D}=\mathbf{e} \boldsymbol{\epsilon}+\boldsymbol{\varepsilon}^{\epsilon} \mathbf{E}
\end{aligned}
$$

where $\mathbf{C}^{\mathrm{E}}, \mathbf{e}, \mathbf{E}, \mathbf{D}, \boldsymbol{\varepsilon}^{\epsilon}$ are the stiffness matrix measured under zero-electric field (short-circuit) condition, piezoelectric stress coefficient matrix, electric field vector, electric displacement vector and permittivity matrix measured at zerostrain (clamped) condition, respectively. Material damping is represented using constant loss factor $\eta[33]$. 
The electric field is assumed to be quasi-static (curl $\mathbf{E}$ is zero) and thus it is expressed as a scalar gradient of the potential [39]

$$
\mathbf{E}(x, y, z)=-\nabla \boldsymbol{\vartheta}(x, y, z)
$$

Given the discretisation of the cross-section one writes [40-42]

$$
\mathbf{E}(x, y, z)=-\left[\begin{array}{lll}
\frac{\partial}{\partial x} & \frac{\partial}{\partial y} & \frac{\partial}{\partial z}
\end{array}\right] \mathbf{N}_{\varphi}(x, y) \boldsymbol{\varphi}(z)
$$

where the electrical potential shape functions $\mathbf{N}_{\varphi}(x, y)$ are chosen to be the same as the displacement shape functions $\mathbf{N}(x, y)$.

In this work piezoelectric elements are developed to be used for modelling actuators made of bulk piezoelectric ceramic (PZT) with two electroded surfaces. A driving voltage is applied across the thickness of the PZT element ( $y$ direction). To enable condensation of the potentials (though it is not necessary), the electric field is assumed not to change along the propagation direction $z$. By doing so, the contribution of the shear piezoelectric coefficient associated with the voltage gradient along $z$ is neglected, which is not expected to invoke a significant error for wave excitation calculation if the corresponding shear deformation in the actuator is relatively small. Eq. (8) is rewritten as:

$$
\mathbf{E}(x, y, z)=-\left[\begin{array}{lll}
\mathbf{N}_{\varphi, x} & \mathbf{N}_{\varphi, y} & 0
\end{array}\right]^{\top} \boldsymbol{\varphi}=-\mathbf{B}_{\varphi} \varphi
$$

Substituting Eqs. (3), (9) into Eq. (6) one gets the constitutive equations expressed in terms of nodal degrees of freedom and element shape functions:

$$
\begin{aligned}
\boldsymbol{\sigma} & =\mathbf{C}^{\mathrm{E}}\left(\mathbf{B}_{1} \mathbf{q}+\mathbf{B}_{2} \frac{\partial}{\partial z} \mathbf{q}\right)+\mathbf{e}^{\top} \mathbf{B}_{\varphi} \boldsymbol{\varphi} \\
\mathbf{D} & =\mathbf{e}\left(\mathbf{B}_{1} \mathbf{q}+\mathbf{B}_{2} \frac{\partial}{\partial z} \mathbf{q}\right)-\boldsymbol{\varepsilon}^{\epsilon} \mathbf{B}_{\varphi} \boldsymbol{\varphi}
\end{aligned}
$$

If one sets the piezoelectric coefficients in $\mathbf{e}$ and dielectric constants in $\varepsilon^{\epsilon}$ to zero, standard elastic Hooke's Law equations are recovered.

\subsection{Virtual work principle for piezoelectric semi-analytical finite element}

The governing equations for wave propagation are obtained with the aid of the virtual work principle for deformable bodies and the analogous principle of 
virtual electric potentials $[39,41,42]$. The analysed section of the waveguide occupies volume $V$ which is formed by a cross-section $\Omega$ extruded through length $L$ and is bounded by surface $\Gamma$. The boundary conditions $(\mathrm{BC})$ on $\Gamma$ are the following:

- Essential mechanical BC on $\Gamma_{u}($ Dirchelet BC): $u=\underline{u}$

- Essential electrical BC on $\Gamma_{\varphi}($ Dirchelet BC): $\varphi=\underline{\varphi}$

- Natural mechanical BC on $\Gamma_{\sigma}\left(\right.$ Neumann BC): $\sigma_{i j} n_{j}=t_{i}$

- Natural electrical BC on $\Gamma_{\varrho}\left(\right.$ Neumann BC): $n_{i} D_{i}=-\varrho_{i}$

where $\underline{u}$ and $\varphi$ are the prescribed displacement and potential, respectively, and $t_{i}, \varrho_{i}$ are the applied surface traction and the applied surface charge, respectively. The bounding surfaces related to each pair of the boundary conditions are exclusive and their unions form the whole surface, i.e. $\Gamma_{u} \cup \Gamma_{\sigma}=\Gamma, \Gamma_{u} \cap \Gamma_{\sigma}=0$ and analogously $\Gamma_{\varphi} \cup \Gamma_{\varrho}=\Gamma, \Gamma_{\varphi} \cap \Gamma_{\varrho}=0$.

Given the constitutive equations, the mechanical equilibrium condition and the essential boundary conditions given above, the virtual work principle states that for every time $t$

$$
\overbrace{\int_{\Gamma_{\sigma}} \delta \mathbf{u}^{\top} \mathbf{t} \mathrm{d} \Gamma_{\sigma}}^{\substack{\text { work done by } \\
\text { external surface } \\
\text { tractions }}}+\overbrace{\delta \mathbf{q}^{\top} \mathbf{f}}^{\begin{array}{c}
\text { work done by } \\
\text { nodal forces }
\end{array}}=\overbrace{\int_{\mathrm{V}} \delta \mathbf{u}^{\top}(\rho \ddot{\mathbf{u}}) \mathrm{d} V}^{\begin{array}{c}
\text { work done by } \\
\text { inertia body forces }
\end{array}}+\overbrace{\int_{\mathrm{V}} \delta \boldsymbol{\epsilon}^{\top} \boldsymbol{\sigma} \mathrm{d} V}^{\begin{array}{c}
\text { work done by } \\
\text { the stress field }
\end{array}}
$$

where $\mathbf{t}$ is the external tractions vector, $\mathbf{f}$ is the vector of nodal forces applied at the ends of the considered section of the waveguide of length $L$ and $\rho$ is the density of the material.

Similarly, under the essential boundary conditions, the virtual electric potentials principle states that for every time $t$

$$
\overbrace{\int_{\Gamma_{\varrho}} \delta \varphi^{\top} \varrho \mathrm{d} \Gamma_{\varrho}}^{\begin{array}{c}
\text { work done by } \\
\text { external surface } \\
\text { charges }
\end{array}}=\overbrace{\int_{\mathrm{V}} \delta \mathbf{E}^{\top} \mathbf{D} \mathrm{d} V}^{\begin{array}{c}
\text { work done by } \\
\text { the electric } \\
\text { displacement field }
\end{array}}
$$


where $\varrho$ is the external surface charge vector.

The constitutive equations from Eq. (10) are substituted into each of the components in Eqs. (11) and (12) along with the finite element approximations given in Eqs. (2), (3) and (9). The detailed algebraic manipulations and integration by parts for all of the components are omitted below for the sake of brevity. To clarify the formulation the following notation is adopted which results from the integration of the matrices over the cross-sectional element domain:

$$
\begin{array}{rlrl}
\mathbf{M} & =\int_{\Omega} \mathbf{N}^{\top} \rho \mathbf{N} \mathrm{d} \Omega & \mathbf{K}_{f} & =\int_{\Omega} \mathbf{B}_{2}^{\top} \mathbf{C B}_{1} \mathrm{~d} \Omega \\
\mathbf{K}_{0}=\int_{\Omega} \mathbf{B}_{1}^{\top} \mathbf{C B}_{1} \mathrm{~d} \Omega & \mathbf{K}_{0}^{u \varphi}=\int_{\Omega} \mathbf{B}_{1}^{\top} \mathbf{e}^{\top} \mathbf{B}_{\varphi} \mathrm{d} \Omega \\
\mathbf{K}_{1}=\int_{\Omega} \mathbf{K}_{f}^{\top}-\mathbf{K}_{f} \mathrm{~d} \Omega & \mathbf{K}_{1}^{u \varphi}=\int_{\Omega} \mathbf{B}_{2}^{\top} \mathbf{e}^{\top} \mathbf{B}_{\varphi} \mathrm{d} \Omega \\
\mathbf{K}_{2}=\int_{\Omega} \mathbf{B}_{2}^{\top} \mathbf{C B}_{2} \mathrm{~d} \Omega & \mathbf{K}^{\varphi \varphi}=\int_{\Omega} \mathbf{B}_{\varphi}^{\top} \boldsymbol{\varepsilon}^{\mathrm{s}} \mathbf{B}_{\varphi} \mathrm{d} \Omega \\
\mathbf{p}=\int_{\Gamma_{\sigma}} \mathbf{N}^{\top} \mathbf{N t}_{i} \mathrm{~d} \Gamma_{\sigma} & \mathbf{\Upsilon} & =\int_{\Gamma_{\varrho}} \boldsymbol{\varrho} \mathrm{d} \Gamma_{\varrho}
\end{array}
$$

Let us now rewrite both Eq. (11) and Eq. (12) using the notation from Eq. (13). Rearranging the terms one obtains

$$
\begin{gathered}
\mathbf{0}=\int_{L}\left\{\delta \mathbf{q}^{\top}\left(\mathbf{M} \frac{\partial^{2}}{\partial t^{2}} \mathbf{q}+\mathbf{K}_{0} \mathbf{q}+\mathbf{K}_{1} \frac{\partial}{\partial z} \mathbf{q}-\mathbf{K}_{2} \frac{\partial^{2}}{\partial z^{2}} \mathbf{q}\right)+\right. \\
\left.\delta \mathbf{q}^{\top}\left(\mathbf{K}_{0}^{u \varphi} \boldsymbol{\varphi}-\mathbf{K}_{1}^{u \varphi} \frac{\partial}{\partial z} \boldsymbol{\varphi}\right)-\delta \mathbf{q}^{\top} \mathbf{p}\right\} \mathrm{d} z+ \\
\delta \mathbf{q}^{\top}\left[\mathbf{K}_{f} \mathbf{q}+\mathbf{K}_{2} \frac{\partial}{\partial z} \mathbf{q}+\mathbf{K}_{1}^{u \varphi} \boldsymbol{\varphi}\right]-\delta \mathbf{q}^{\top} \mathbf{f}
\end{gathered}
$$

and

$$
\mathbf{0}=\int_{L}\left\{\delta \boldsymbol{\varphi}^{\top} \mathbf{K}^{\varphi \varphi} \boldsymbol{\varphi}-\delta \boldsymbol{\varphi}^{\top}\left(\mathbf{K}_{0}^{\varphi u} \mathbf{q}+\mathbf{K}_{1}^{\varphi u} \frac{\partial}{\partial z} \mathbf{q}\right)-\delta \boldsymbol{\varphi}^{\top} \boldsymbol{\Upsilon}\right\} \mathrm{d} z
$$

Eq. (14) contains two main terms - the first is related to the wave field behaviour over the length $L$ and the second corresponds to cross-sections at the ends of $L$. Both equations need to hold for every time $t$ and any admissible virtual 
displacement $\delta q$ and virtual potential $\delta \varphi$, which leads to the governing equation for wave propagation in an elastic waveguide with piezoelectric coupling.

\subsection{Governing equations for wave propagation and resultant nodal forces}

From Eqs. (14), (15) one can conclude the set of the governing equations for wave propagation,

$$
\begin{array}{r}
\mathbf{M} \frac{\partial^{2} \mathbf{q}}{\partial t^{2}}+\mathbf{K}_{0} \mathbf{q}+\mathbf{K}_{1} \frac{\partial \mathbf{q}}{\partial z}-\mathbf{K}_{2} \frac{\partial^{2} \mathbf{q}}{\partial z^{2}}+\mathbf{K}_{0}^{u \varphi} \boldsymbol{\varphi}-\mathbf{K}_{1}^{u \varphi} \frac{\partial \varphi}{\partial z}=\mathbf{p} \\
\mathbf{K}_{0}^{\varphi u} \mathbf{q}+\mathbf{K}_{1}^{\varphi u} \frac{\partial \mathbf{q}}{\partial z}-\mathbf{K}^{\varphi \varphi} \boldsymbol{\varphi}=-\mathbf{\Upsilon}
\end{array}
$$

and the natural boundary condition stating that at the ends of the considered section of the waveguide

$$
\mathbf{q}=0 \quad \text { or } \quad \mathbf{f}_{i}=\mathbf{K}_{f} \mathbf{q}+\mathbf{K}_{2} \frac{\partial \mathbf{q}}{\partial z}+\mathbf{K}_{1}^{u \varphi} \boldsymbol{\varphi}
$$

which directly provides the expression for the resultant nodal forces. Solution to Eq. (16) is performed using the Spatial Fourier Transform (SFT) defined as

$$
\mathcal{F}\{\mathbf{q}\}=\overline{\mathbf{q}}=\int_{-\infty}^{\infty} \mathbf{q e}^{\jmath k z} \mathrm{~d} z \quad \text { and } \mathbf{q}=\frac{1}{2 \pi} \int_{-\infty}^{\infty} \overline{\mathbf{q}} \mathrm{e}^{-\jmath k z} \mathrm{~d} k
$$

which yields that the SFT of the space derivative of a quantity is

$$
\mathcal{F}\left\{\frac{\partial}{\partial z} \mathbf{q}\right\}=-\jmath k \mathcal{F}\{\mathbf{q}\}=-\jmath k \overline{\mathbf{q}}
$$

Applying Eqs. (18) and (19) to Eq. (16) and assuming time harmonic motion assumption, one writes

$$
\left(\left[\begin{array}{cc}
-\omega^{2} \mathbf{M} & \mathbf{0} \\
\mathbf{0} & \mathbf{0}
\end{array}\right]+\left[\begin{array}{cc}
\mathbf{K}_{0}-\jmath k \mathbf{K}_{1}-(-\jmath k)^{2} \mathbf{K}_{2} & \mathbf{K}_{0}^{u \varphi}+\jmath k \mathbf{K}_{1}^{u \varphi} \\
\mathbf{K}_{0}^{\varphi u}-\jmath k \mathbf{K}_{1}^{\varphi u} & -\mathbf{K}^{\varphi \varphi}
\end{array}\right]\right) \cdot\left\{\begin{array}{c}
\overline{\mathbf{q}} \\
\overline{\boldsymbol{\varphi}}
\end{array}\right\}=\left\{\begin{array}{c}
\overline{\mathbf{p}} \\
-\overline{\mathbf{\Upsilon}}
\end{array}\right\}
$$

Eq. (20) is the governing equation for wave propagation in a waveguide with piezoelectric coupling in the wavenumber-frequency domain. After the same transformations the resultant forces over the cross-section are given by

$$
\overline{\mathbf{f}}=\mathbf{K}_{f} \overline{\mathbf{q}}-\jmath k \mathbf{K}_{2} \overline{\mathbf{q}}+\mathbf{K}_{1}^{u \varphi} \bar{\varphi}
$$




\subsection{Static condensation of the internal potentials}

Typical piezoceramic actuators are electroded on the surfaces perpendicular to the thickness direction. One of the electrodes is grounded and the other is driven. It may therefore be advantageous to express the above equation in terms of the driving voltage which can be done by condensing out the potentials at the internal nodes via static condensation $[40,41,43]$. The procedure is described in detail in the Appendix. The governing equation becomes

$$
\left[\begin{array}{cc}
\tilde{\mathbf{K}}_{0}-\jmath k \tilde{\mathbf{K}}_{1}-(-\jmath k)^{2} \tilde{\mathbf{K}}_{2}-\omega^{2} \mathbf{M} & \tilde{\mathbf{K}}_{0}^{u \varphi}+\jmath k \tilde{\mathbf{K}}_{1}^{u \varphi} \\
\tilde{\mathbf{K}}_{0}^{\varphi u}-\jmath k \tilde{\mathbf{K}}_{1}^{\varphi u} & -\tilde{\mathbf{K}}^{\varphi \varphi}
\end{array}\right] \cdot\left\{\begin{array}{c}
\overline{\mathbf{q}} \\
V
\end{array}\right\}=\left\{\begin{array}{c}
\overline{\mathbf{p}} \\
-Q
\end{array}\right\}
$$

where $Q$ is the total charge over the powered electrode and $V$ is the amplitude of the time-harmonic driving voltage. The tilde $\{\tilde{\}}$ symbol indicates that the matrix is amended to account for the condensed potentials.

Analogously, the internal forces vector is rewritten as

$$
\overline{\mathbf{f}}=\tilde{\mathbf{K}}_{f} \overline{\mathbf{q}}-\jmath k \tilde{\mathbf{K}}_{2} \overline{\mathbf{q}}+\tilde{\mathbf{K}}_{1}^{u \varphi} V
$$

Although condensation simplifies the notation and the solution procedure, it also involves the loss of sparsity of SAFE matrices. Therefore, from a numerical performance viewpoint condensation may not be the optimal choice. Moreover, static condensation requires neglecting the shear piezoelectric coefficient (see Sec. 2.2). This, in some cases may lead to inaccurate prediction. If static condensation is not desired, the solution procedure outlined below remains the same, but it is performed on the original set of equations.

\section{Free elastic waves in a waveguide with piezoelectric coupling}

Let us now look at free wave propagation in an elastic waveguide with piezoelectric coupling. As a consequence of the quasi-electrostatic assumption, only elastic waves are permitted. The role of the piezoelectric coupling is to provide means of exciting the waveguide, sensing the travelling waves or harvesting the mechanical energy. Before the response to the distributed voltage is introduced we look at the calculation and basic characteristics of the free waves which form a basis for the forced response solution. 


\subsection{Electrical conditions}

Free waves in a waveguide with piezoelectric coupling can be considered either in the short-circuit or in the open-circuit condition. The former implies that the voltage in the powered electrode is set to zero $(v=0)$ resulting in the quadratic eigenvalue problem (QEP)

$$
\left[\tilde{\mathbf{K}}_{0}+\lambda \tilde{\mathbf{K}}_{1}-\lambda^{2} \tilde{\mathbf{K}}_{2}-\omega^{2} \mathbf{M}\right] \boldsymbol{\phi}=\mathbf{0}
$$

where $\phi$ is the eigenvector and $\lambda=-\jmath k$.

Conversely, for the open-circuit electrodes the 'driving' charge $Q$ is set to zero. The modal potential at the powered electrode is extracted from the second

row of Eq.(22), i.e. $V=\left(\tilde{\mathbf{K}}^{\varphi \varphi}\right)^{-1}\left(\tilde{\mathbf{K}}_{0}^{\varphi u}-\jmath k \tilde{\mathbf{K}}_{1}^{\varphi u}\right) \phi$ and substituted back in the first row equation. After some manipulation one obtains

$$
\begin{aligned}
& \left\{\left[\tilde{\mathbf{K}}_{0}+\tilde{\mathbf{K}}_{0}^{u \varphi}\left(\tilde{\mathbf{K}}^{\varphi \varphi}\right)^{-1} \tilde{\mathbf{K}}_{0}^{\varphi u}-\omega^{2} \mathbf{M}\right]+\right. \\
& \lambda\left[\tilde{\mathbf{K}}_{1}+\tilde{\mathbf{K}}_{0}^{u \varphi}\left(\tilde{\mathbf{K}}^{\varphi \varphi}\right)^{-1} \tilde{\mathbf{K}}_{1}^{\varphi u}-\tilde{\mathbf{K}}_{1}^{u \varphi}\left(\tilde{\mathbf{K}}^{\varphi \varphi}\right)^{-1} \tilde{\mathbf{K}}_{0}^{\varphi u}\right]- \\
& \left.\lambda^{2}\left[\tilde{\mathbf{K}}_{2}+\tilde{\mathbf{K}}_{1}^{u \varphi}\left(\tilde{\mathbf{K}}^{\varphi \varphi}\right)^{-1} \tilde{\mathbf{K}}_{1}^{\varphi u}\right]\right\} \phi=0
\end{aligned}
$$

The form of the eigenvalue problem is similar to Eq. (24) but the stiffness matrices are enhanced. Therefore, Eq. (25) indicates that in the open-circuit condition the structure is stiffened compared to the short-circuit case. This is a well-known effect of the piezoelectric coupling on the elastic behaviour of the material.

In this paper we consider the voltage to be the 'driving' variable. The charge is a response variable associated with how the actuator-structure system responds to the applied voltage. Therefore, the short-circuit condition equations are considered hereafter.

\subsection{Free wave solution}

Solution to the QEP in Eq. (24) can be performed using any available numerical approach [44]. The most common is companion matrix linearisation. 
A new eigenvector is introduced as $\hat{\phi}=\left[\begin{array}{ll}\phi & \lambda \phi\end{array}\right]^{\top}$ and the SAFE governing equation from Eq. (24) is rewritten as a generalised eigenvalue problem (GEP)

$$
\left(\left[\begin{array}{cc}
\mathbf{0} & \omega^{2} \mathbf{M}-\tilde{\mathbf{K}}_{0} \\
\omega^{2} \mathbf{M}-\tilde{\mathbf{K}}_{0} & \tilde{\mathbf{K}}_{1}
\end{array}\right]-\lambda\left[\begin{array}{cc}
\omega^{2} \mathbf{M}-\tilde{\mathbf{K}}_{0} & \mathbf{0} \\
\mathbf{0} & \tilde{\mathbf{K}}_{2}
\end{array}\right]\right)\left\{\begin{array}{c}
\boldsymbol{\phi} \\
\lambda \phi
\end{array}\right\}=\mathbf{0}
$$

or more concisely as

$$
[\mathbf{A}(\omega)-\lambda \mathbf{B}(\omega)] \hat{\boldsymbol{\phi}}=\mathbf{0}
$$

where $\mathbf{A}, \mathbf{B}$ are of dimensions $2 n \times 2 n, n$ is the number of the displacement degrees of freedom in the cross-section.

The GEP from Eq. (27) can be readily solved using any of the common scientific computing packages. For a given frequency $\omega$ one finds the set of $2 n$ eigenvalues $\boldsymbol{\Lambda}$ (associated with $n$ positive- and $n$ negative-going waves), and $2 n \times 2 n$ matrices of the corresponding right and left eigenvectors denoted by $\hat{\boldsymbol{\Phi}}$ and $\hat{\boldsymbol{\Psi}}$, respectively. The right eigenvectors $\hat{\boldsymbol{\phi}}_{i}$ are columns of $\hat{\boldsymbol{\Phi}}$ and left eigenvectors $\hat{\boldsymbol{\psi}}_{i}$ are rows of $\hat{\boldsymbol{\Psi}}$.

The wavenumbers can be extracted from the eigenvalues since $k=\jmath \lambda$. The wave is considered to be positive-going if [45]:

$$
\left|\mathrm{e}^{-\jmath k_{i} \Delta}\right|<1 \quad \text { or } \quad\left|\mathrm{e}^{-\jmath k_{i} \Delta}\right|=1 \quad \text { and } \quad \Pi_{i}>0
$$

where $\Delta$ is a distance smaller than the smallest wavelength of interest and $\Pi_{i}$ is the wave power flow along the propagation direction adopted from the mechanical complex Poynting theorem [36]. Wave power flow is calculated in SAFE as [46]

$$
\Pi_{i}=\omega \frac{\operatorname{Im}\left\{\phi_{i}^{* \top}\left[\tilde{\mathbf{K}}_{f}-\jmath k_{i} \tilde{\mathbf{K}}_{2}\right] \boldsymbol{\phi}_{i}\right\}}{2}
$$

The left and right eigenvectors are normalised according to their orthogonality with respect to the $\mathbf{B}$ matrix (assuming that $\mathbf{B}$ is not rank deficient), so that

$$
\hat{\boldsymbol{\psi}}_{i} \mathbf{B}(\omega) \hat{\boldsymbol{\phi}}_{j}\left\{\begin{array}{ll}
1 & \text { if } i=j \\
0 & \text { otherwise }
\end{array} \text { and } \quad \hat{\boldsymbol{\psi}}_{i} \mathbf{A}(\omega) \hat{\boldsymbol{\phi}}_{i}=\lambda_{i}\right.
$$




\subsection{Wave basis}

The SAFE formulation is used to obtain the characteristics of the waveguide (wavenumbers and mode shapes) which are called a wave basis [47, 48]. The nodal displacements and nodal resultant forces at any location in the waveguide are expressed in terms of the wave basis

$$
\left\{\begin{array}{l}
\mathbf{q}(z) \\
\mathbf{f}(z)
\end{array}\right\}=\left\{\begin{array}{l}
\boldsymbol{\Phi} \\
\boldsymbol{\Theta}
\end{array}\right\} \mathbf{a}(z)=\left[\begin{array}{ll}
\boldsymbol{\phi}^{+} & \boldsymbol{\phi}^{-} \\
\boldsymbol{\theta}^{+} & \boldsymbol{\theta}^{-}
\end{array}\right]\left\{\begin{array}{l}
\mathbf{a}^{+}(z) \\
\mathbf{a}^{-}(z)
\end{array}\right\}
$$

where $\boldsymbol{\phi}^{+,-}$are positive- and negative-going displacement wave mode shapes ob-

tained by taking the upper half of the extended eigenvector $\hat{\boldsymbol{\phi}}, \boldsymbol{\theta}^{+,-}$are the force wave mode shapes, and $\mathbf{a}^{+}(z), \mathbf{a}^{-}(z)$ are the corresponding wave amplitudes. The force wave mode shapes are obtained from Eq. (23):

$$
\boldsymbol{\theta}=\left[\begin{array}{ll}
\tilde{\mathbf{K}}_{f} & \tilde{\mathbf{K}}_{2}
\end{array}\right] \hat{\boldsymbol{\phi}}
$$

The change of the wave amplitude along the waveguide is described by the space-harmonic term called the propagation matrix $\boldsymbol{\tau}(z)=\operatorname{diag}\left(\mathrm{e}^{-\jmath k_{i} z}\right)$.

\section{Excitation of structural waves in an elastic waveguide covered with a piezoelectric layer powered over a finite length}

In this section we calculate structural waves excitation as a response to a spatially distributed voltage using the piezoelectric wave element developed above. We consider an infinite elastic layer covered over its whole length by an electroded piezoelectric layer (Fig. 2). A finite section of the electrode is driven with a time-harmonic voltage $v_{0}(t)$ (dotted region). The 'driving' section is electrically isolated from adjacent parts of the waveguide which are in the short-circuit condition. Vectors $\mathbf{p}^{-}$and $\mathbf{p}^{+}$represent the waves generated by the distributed patch-like piezoelectric excitation as they enter the non-powered region. A local coordinate system $\left(z^{\prime}\right)$ is introduced with the origin in the middle of the length of the piezoelectric actuator. The spatial distribution of voltage 


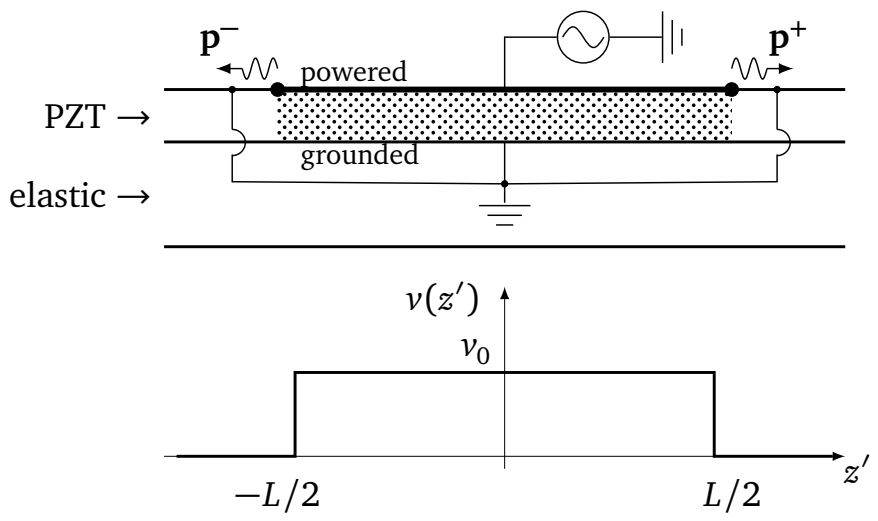

Figure 2: Elastic waveguide covered by a piezoelectric layer powered over a finite length. Excited waves amplitudes and voltage spatial distribution.

$v\left(z^{\prime}, t\right)$ is a rectangular box function as shown in Fig. 2, such that

$$
v\left(z^{\prime}, t\right)= \begin{cases}v_{0}(t)=V_{0} \mathrm{e}^{\jmath \omega t} & \text { if } z^{\prime} \leq|L / 2| \\ 0 & \text { otherwise }\end{cases}
$$

The time-harmonic term in the driving voltage is dropped for brevity hereafter.

The wave amplitudes can be found by solving the SAFE equation in the wavenumber-frequency domain directly, given the spatial distribution of voltage (this approach is similar to [48] where the response to a distributed pressure is considered).

Let us recall the top row of the governing equation for wave propagation in the waveguide with piezoelectric coupling in Eq. (22) and assume no external tractions for the sake of clarity of the formulation:

$$
\left[\tilde{\mathbf{K}}_{0}-\jmath k \tilde{\mathbf{K}}_{1}-(-\jmath k)^{2} \tilde{\mathbf{K}}_{2}-\omega^{2} \mathbf{M}\right] \overline{\mathbf{q}}=\left(-\tilde{\mathbf{K}}_{0}^{u \varphi}-\jmath k \tilde{\mathbf{K}}_{1}^{u \varphi}\right) \bar{V}_{0}
$$

where $\bar{V}_{0}$ is the SFT of the driving voltage spatial distribution given by

$$
\bar{V}_{0}=2 V_{0} \frac{\sin (k L / 2)}{k}
$$

The solution is performed on the linearised form of Eq.(34) which enables 
the use of GEP properties:

$$
[\mathbf{A}(\omega)-(-\jmath k) \mathbf{B}(\omega)] \overline{\mathbf{q}}=\left[\begin{array}{c}
0 \\
-\tilde{\mathbf{K}}_{0}^{u \varphi}-\jmath k \tilde{\mathbf{K}}_{1}^{u \varphi}
\end{array}\right] \bar{V}_{0}
$$

The right hand side matrix in Eq. (36) is for brevity denoted as $\mathbf{H}$ hereafter. The displacement vector $\overline{\mathbf{q}}$ can be written as an expansion in terms of eigenvectors, that is as a weighted superposition of the travelling waves, i.e. $\overline{\mathbf{q}}=\sum_{i=1}^{2 n} \hat{\phi}_{i} \bar{p}_{i}$. After pre-multiplying by $\hat{\boldsymbol{\psi}}_{\mathbf{i}}$, exploiting the orthogonality of the eigenvectors with respect to $\mathbf{B}$ (Eq. (30)) and substituting $\bar{V}_{0}$ with Eq. (35), one writes the nodal displacement solution

$$
\bar{p}_{i}=\frac{2 V_{0} \sin (k L / 2)}{\jmath k\left(k-k_{i}\right)} \hat{\boldsymbol{\psi}}_{\mathbf{i}} \mathbf{H}
$$

The modal weighting factor representing the amplitude of wave $i$ in the space domain is found via the inverse SFT defined in Eq. (18):

$$
p_{i}\left(z^{\prime}\right)=\frac{1}{2 \pi} \int_{-\infty}^{+\infty} \bar{p}_{i} \mathrm{e}^{-\jmath k z^{\prime}} \mathrm{d} k=\frac{1}{2 \pi} \int_{-\infty}^{+\infty} \frac{2 V_{0} \sin (k L / 2)}{\jmath k\left(k-k_{i}\right)} \hat{\boldsymbol{\psi}}_{\mathbf{i}} \mathbf{H e}^{-\jmath k z^{\prime}} \mathrm{d} k
$$

The above complex integral has simple poles at $k_{i}$ and a removable singularity at $k=0$ (since the limit for $k$ approaching 0 is 1 ), therefore it is evaluated using Cauchy's residue theorem [49]. The choice of the contour of integration depends on the solutions of interest. In the case of an undamped waveguide the contours need to be properly amended to classify the purely real wavenumbers appropriately(see Fig. 3).

Given the notation from Fig. 3, the Cauchy residue theorem states that [49]

$$
\begin{aligned}
& \int_{-\infty}^{+\infty} F(k) \mathrm{e}^{-\jmath k z^{\prime}} \mathrm{d} k=
\end{aligned}
$$

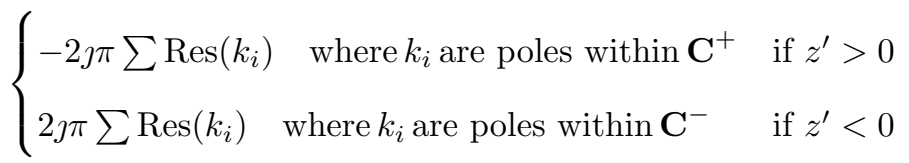

where the residues are calculated as [50]

$$
\operatorname{Res}\left(\frac{a(k)}{b(k)}, k_{0}\right)=\frac{a\left(k_{0}\right)}{\left.\frac{\mathrm{d} b(k)}{\mathrm{d} k}\right|_{k=k_{0}}}
$$




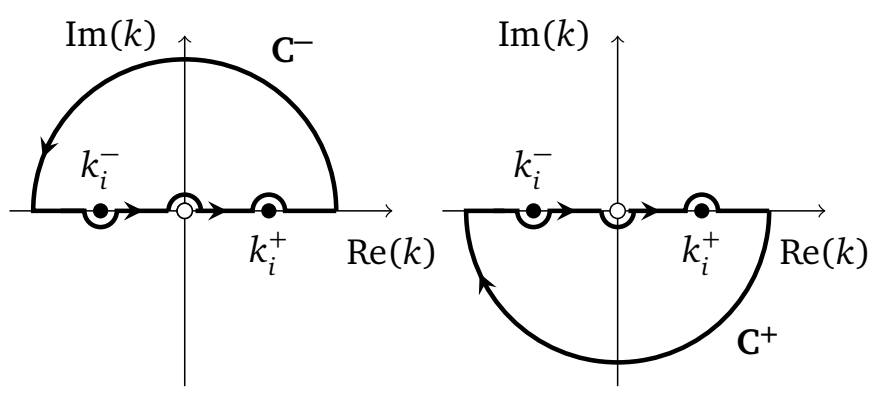

Figure 3: Contours for integration [48].

After evaluating the integral one obtains the excited wave amplitudes in the space domain:

$$
\begin{aligned}
& p_{i}^{+}\left(z^{\prime}\right)=-\frac{2 V_{0} \sin \left(k_{i}^{+} L / 2\right)}{k_{i}^{+}} \hat{\boldsymbol{\psi}}_{\mathbf{i}}^{+} \mathbf{H e}^{-\jmath k_{i}^{+} z^{\prime}} \\
& p_{i}^{-}\left(z^{\prime}\right)=\frac{2 V_{0} \sin \left(k_{i}^{-} L / 2\right)}{k_{i}^{-}} \hat{\boldsymbol{\psi}}_{\mathbf{i}}^{-} \mathbf{H e}^{-\jmath k_{i}^{-} z^{\prime}}
\end{aligned}
$$

Note that $p_{i}^{+}\left(z^{\prime}\right), p_{i}^{-}\left(z^{\prime}\right)$ are expressed with reference to the local coordinate system as depicted in Fig. 2. To obtain the excited wave amplitudes as they appear at the ends of the electrically driven region, they are evaluated at $x=$ $\pm L / 2$, yielding:

$$
\begin{aligned}
& p_{i}^{+}=-\frac{V_{0}\left(1-\mathrm{e}^{-\jmath k_{i}^{+} L}\right)}{\jmath k_{i}^{+}} \hat{\boldsymbol{\psi}}_{\mathbf{i}}^{+} \mathbf{H} \\
& p_{i}^{-}=\frac{V_{0}\left(\mathrm{e}^{\jmath k_{i}^{-} L}-1\right)}{\jmath k_{i}^{-}} \hat{\boldsymbol{\psi}}_{\mathbf{i}}^{-} \mathbf{H}
\end{aligned}
$$

Eq. (42) provides expressions for wave amplitudes excited by a voltage uniformly distributed over $L$ in an infinite piezo-covered waveguide as they appear at the ends of the driving section.

\section{Excitation of structural waves with a finite piezoelectric actuator}

In this section the tools outlined previously are put together to form a coupled electro-mechanical wave model. An illustrative example of a beam-like waveguide (2D rectangular cross-section) with a PZT actuator is presented in detail, however the framework can be applied to an arbitrary multi-component 
(a)

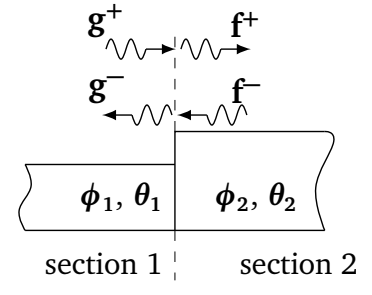

(b)

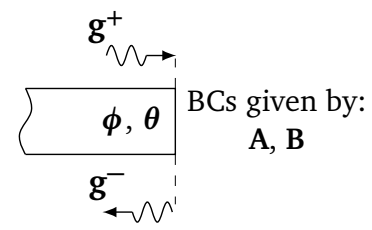

Figure 4: (a) Wave scattering at a junction between two waveguides; (b) Wave reflection at the boundary.

structural waveguide with piezoelectric actuators [51]. The chosen example shows the technique's capability of predicting a 3D wave field. Lamb waves can be simulated if the cross-section is modelled using mono-dimensional plane strain elements.

The proposed algorithm can be summarised in the following steps: the structure is first divided into sections that have homogeneous properties along the propagation direction (wave elements); for each of the wave elements the wave bases and excited wave amplitudes are obtained using the SAFE methodology outlined above; afterwards, the coupling between the wave elements is implemented using scattering matrices which describe how the waves behave when incident upon discontinuities (e.g. junctions between different wave elements); the final response is calculated using wave propagation/scattering relationships expressed in matrix form.

\subsection{Wave scattering and reflection}

The wave approach involves coupling together wave elements that have different dimensions and properties. The wave elements are required to be meshed in such a way that all the nodes from the overlapping regions of two adjacent waveguides are coincident. Wave scattering at discontinuities and reflection at the boundaries is represented here in the same way as proposed in $[47,52]$.

When incident on a junction, the wave scatters into reflected and transmitted components. The frequency dependent scattering matrix can be found by 
solving simultaneously the conditions of displacement continuity and force equilibrium between the adjacent wave elements [52]. With reference to Fig. 4, let us write them in matrix form using the wave basis defined in Eq. (31)

$$
\left[\begin{array}{cc}
-\mathbf{C}_{1} \boldsymbol{\phi}_{1}^{-} & \mathbf{C}_{2} \boldsymbol{\phi}_{2}^{+} \\
-\mathbf{E}_{1} \boldsymbol{\theta}_{1}^{-} & \mathbf{E}_{2} \boldsymbol{\theta}_{2}^{+}
\end{array}\right]\left\{\begin{array}{l}
\mathrm{g}^{-} \\
\mathbf{f}^{+}
\end{array}\right\}=\left[\begin{array}{ll}
\mathbf{C}_{1} \phi_{1}^{+} & -\mathbf{C}_{2} \phi_{2}^{-} \\
\mathbf{E}_{1} \boldsymbol{\theta}_{1}^{+} & -\mathbf{E}_{2} \boldsymbol{\theta}_{2}^{-}
\end{array}\right]\left\{\begin{array}{l}
\mathrm{g}^{+} \\
\mathbf{f}^{-}
\end{array}\right\}
$$

where $\mathbf{C}_{1}, \mathbf{C}_{2}$ are continuity matrices that provide information about the coincident nodes in neighbouring wave elements and leaving the uncoupled nodes free, whereas $\mathbf{E}_{1}, \mathbf{E}_{2}$ are equilibrium matrices that are balancing the nodal resultant forces at the coincident nodes and setting the resultant nodal forces at the uncoupled nodes to zero.

By multiplying Eq. (43) by the inverse of the matrix on the left hand side, one can express the waves leaving the junction in terms of the waves incident upon the junction by means of the scattering matrix $\mathbf{T}$ :

$$
\left\{\begin{array}{l}
\mathbf{g}^{-} \\
\mathbf{f}^{+}
\end{array}\right\}=\left[\begin{array}{ll}
\mathbf{R}^{11} & \mathbf{T}^{21} \\
\mathbf{T}^{12} & \mathbf{R}^{22}
\end{array}\right]\left\{\begin{array}{l}
\mathbf{g}^{+} \\
\mathbf{f}^{-}
\end{array}\right\}=\mathbf{T}\left\{\begin{array}{l}
\mathbf{g}^{+} \\
\mathbf{f}^{-}
\end{array}\right\}
$$

where the structure of the scattering matrix is shown clearly.

Similarly, wave reflection at a boundary can be determined using the wave basis. Depending on the type of the boundary condition either the displacement or the force degree of freedom is set to zero. With reference to Fig. 4 this can be represented in a matrix form as [47]

$$
\mathbf{A f}+\mathbf{B q}=0
$$

which in terms of the wave basis defined in Eq. (31) is written as

$$
\mathbf{A}\left[\boldsymbol{\theta}^{+} \mathbf{g}^{+}+\boldsymbol{\theta}^{-} \mathbf{g}^{-}\right]+\mathbf{B}\left[\boldsymbol{\phi}^{+} \mathbf{g}^{+}+\boldsymbol{\phi}^{-} \mathbf{g}^{-}\right]=0
$$

The reflection matrix relates the wave leaving the boundary to the wave incident upon the boundary, such that $\mathbf{g}^{-}=\mathbf{R g}^{+}$(for the 'right' boundary). After rearranging Eq. (46) one obtains an expression for a reflection matrix at the 'right' boundary, i.e. with positive-going wave incident, given the characteristics of the boundary $\mathbf{A}, \mathbf{B}$

$$
\mathbf{R}=-\left[\mathbf{A} \boldsymbol{\theta}^{-}+\mathbf{B} \boldsymbol{\phi}^{-}\right]^{-1}\left[\mathbf{A} \boldsymbol{\theta}^{+}+\mathbf{B} \boldsymbol{\phi}^{+}\right]
$$




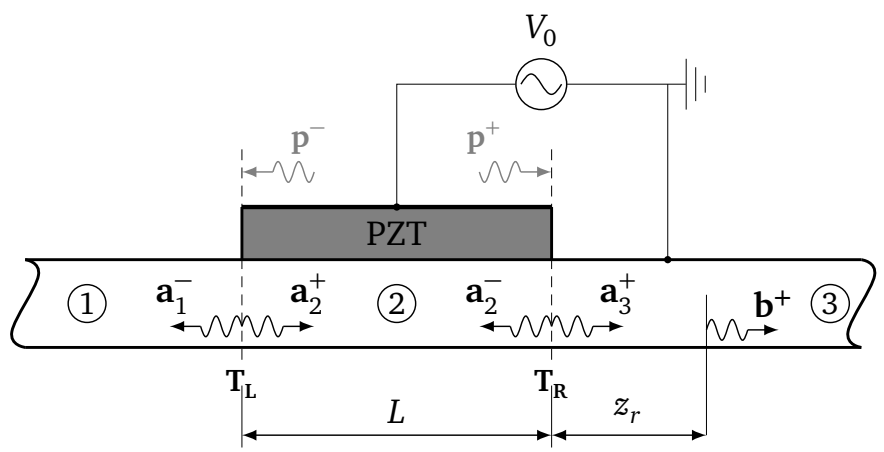

Figure 5: Infinite waveguide excited with a piezoelectric actuator - schematic diagram for calculating the travelling waves outside the PZT-covered section; the excited waves $\mathbf{p}^{+}, \mathbf{p}^{-}$ are defined at the edges of the actuator just before they are incident on the discontinuity; $\mathbf{a}_{i}^{+}$, $\mathbf{a}_{i}^{-}$are the travelling waves at different stages.

\subsection{Example: an infinite beam-like waveguide with a piezoelectric actuator}

The approach is demonstrated on a simple model of an infinite waveguide with a rectangular cross-section equipped with a finite piezo actuator. There are two possible desired outputs of such a calculation, namely either the excited wave field propagating in the elastic substrate or the electrical impedance of the actuator. Both cases are considered below.

\subsubsection{The response outside the piezo-covered region}

Of interest is finding the wave amplitudes induced in the substrate waveguide at a position $z_{r}$ to the right of the actuator, denoted hereafter by $\mathbf{b}^{+}$. The travelling waves at different stages and the excited waves at the ends of the actuator are depicted in Fig. 5.

The wave amplitudes resulting from the distributed voltage excitation $\mathbf{p}^{-}$, $\mathbf{p}^{+}$as found in Sec. 4 are immediately incident upon the discontinuities at the ends of the piezoelectric actuator, and therefore scattered according to the appropriate scattering matrices for the junctions.

The right boundary condition is ideally absorbing (located at $+\infty$ ), so there is no negative-going wave in element 3 . The response of the structure at $z_{r}$ is 
written in the wave domain as

$$
\mathbf{q}\left(z_{r}\right)=\boldsymbol{\phi}^{+} \mathbf{b}^{+}\left(z_{r}\right)=\boldsymbol{\phi}^{+} \boldsymbol{\tau}\left(z_{r}\right) \mathbf{a}_{3}^{+}
$$

To obtain the response at a desired location $z_{r}$, one needs to find the travelling wave amplitude $\mathbf{a}_{\mathbf{3}}{ }^{+}$first

$$
\mathbf{a}_{3}^{+}=\mathbf{T}_{\mathrm{R}}^{12} \mathbf{p}^{+}+\mathbf{T}_{\mathrm{R}}^{12} \boldsymbol{\tau}(L) \mathbf{a}_{2}^{+}
$$

where

$$
\begin{aligned}
& \mathbf{a}_{2}^{+}=\mathbf{R}_{\mathrm{L}}^{22} \mathbf{p}^{-}+\mathbf{R}_{\mathrm{L}}^{22} \boldsymbol{\tau}(L) \mathbf{a}_{2}^{-} \\
& \mathbf{a}_{2}^{-}=\mathbf{R}_{\mathrm{R}}^{11} \mathbf{p}^{+}+\mathbf{R}_{\mathrm{R}}^{11} \boldsymbol{\tau}(L) \mathbf{a}_{2}^{+}
\end{aligned}
$$

Appropriate manipulation of the above equations leads to

$$
\begin{aligned}
\mathbf{a}_{3}^{+}=\mathbf{T}_{\mathrm{R}}^{12} \mathbf{p}^{+}+\mathbf{T}_{\mathrm{R}}^{12} \boldsymbol{\tau}(L)\left[\mathbf{I}-\mathbf{R}_{\mathrm{L}}^{22} \boldsymbol{\tau}(L) \mathbf{R}_{\mathrm{R}}^{11} \boldsymbol{\tau}(L)\right]^{-1} . \\
\\
\quad\left[\mathbf{R}_{\mathrm{L}}^{22} \mathbf{p}^{-}+\mathbf{R}_{\mathrm{L}}^{22} \boldsymbol{\tau}(L) \mathbf{R}_{\mathrm{R}}^{11} \mathbf{p}^{+}\right]
\end{aligned}
$$

which after substituting into Eq. (48) gives the desired response.

5.2.2. The response within the piezo-covered region: electrical impedance extraction

Many engineering applications take advantage of the electromechanical coupling in piezoelectric elements to sense the dynamic behaviour of a structure by means of a frequency dependent electrical impedance of the PZT element $Z(\omega)=\frac{V(\omega)}{I(\omega)}$.

The driving voltage $V_{0}$ is kept fixed, whereas the current drawn is obtained from the second row of Eq. (22) which provides an expression for the total reaction charge on the electrode:

$$
\left[\begin{array}{ll}
\tilde{\mathbf{K}}_{0}^{\varphi u}-\jmath k \tilde{\mathbf{K}}_{1}^{\varphi u} & -\tilde{\mathbf{K}}^{\varphi \varphi}
\end{array}\right]\left\{\begin{array}{c}
\overline{\mathbf{q}} \\
V_{0}
\end{array}\right\}=-Q
$$

The total charge consists of two parts: the component resulting from the electrical properties of the piezoelectric $\left(\tilde{\mathbf{K}}^{\varphi \varphi}\right)$ which has a capacitive nature, and the component resulting from the mechanical deformation of the element generating charge via piezoelectric coupling $\left(\tilde{\mathbf{K}}_{0}^{\varphi u}-\jmath k \tilde{\mathbf{K}}_{1}^{\varphi u}\right)$. 


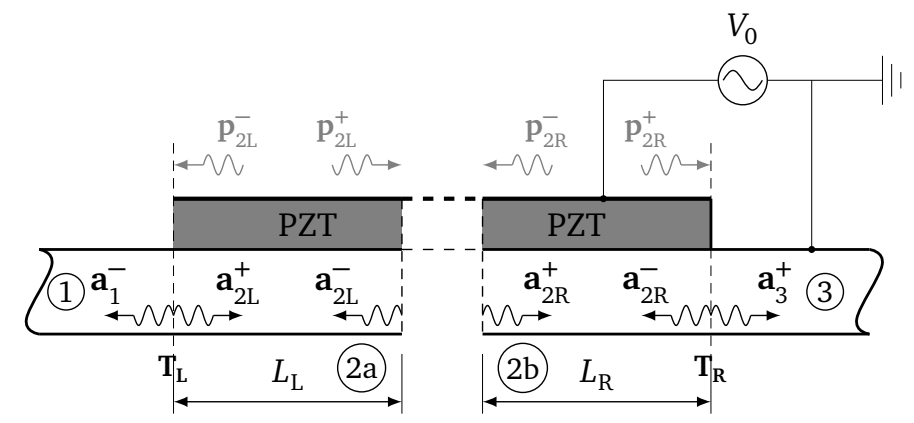

Figure 6: Infinite waveguide excited with a piezoelectric actuator - schematic diagram for calculating the travelling waves inside the PZT-covered section; the excited waves $\mathbf{p}_{2 \mathrm{~L}}^{+}, \mathbf{p}_{2 \mathrm{~L}}^{-}, \mathbf{p}_{2 \mathrm{R}}^{+}$, $\mathbf{p}_{2 \mathrm{R}}^{-}$are defined at the edges of the actuator just before they are incident on the discontinuity; $\mathbf{a}_{i}^{+}, \mathbf{a}_{i}^{-}$are the travelling waves at different stages.

The reaction charge needs to be summed over the length of the actuator, hence the response inside the actuator is required. At each location $z_{i}$ along the length of the PZT-covered wave element we split it into two parts: the one to the left from $z_{i}$ and that to the right from $z_{i}$. The excited waves are calculated separately for each part using the framework presented in the previous paragraphs and substituting the correct lengths depending on $z_{i}$. A schematic diagram for the calculation is shown in Fig. 6.

One starts the calculation by writing the expressions for travelling waves at different stages

$$
\begin{array}{ll}
\mathbf{a}_{2 R}^{+}=\mathbf{p}_{2 L}^{+}+\boldsymbol{\tau}\left(L_{L}\right) \mathbf{a}_{2 L}^{+} & \mathbf{a}_{2 R}^{-}=\mathbf{R}_{R}^{11} \mathbf{p}_{2 R}^{+}+\mathbf{R}_{R}^{11} \boldsymbol{\tau}\left(L_{R}\right) \mathbf{a}_{2 R}^{+} \\
\mathbf{a}_{2 L}^{-}=\mathbf{p}_{2 R}^{-}+\boldsymbol{\tau}\left(L_{R}\right) \mathbf{a}_{2 R}^{-} & \mathbf{a}_{2 L}^{+}=\mathbf{R}_{L}^{22} \mathbf{p}_{2 L}^{-}+\mathbf{R}_{L}^{22} \boldsymbol{\tau}\left(L_{L}\right) \mathbf{a}_{2 L}^{-}
\end{array}
$$

After some algebraic manipulations, one obtains the expressions for the positiveand negative-going waves at a location $z_{i}$ within the piezoelectric actuator

$$
\begin{aligned}
\mathbf{a}_{2 L}^{-}=\left[\mathbf{I}-\boldsymbol{\tau}\left(L_{R}\right) \mathbf{R}_{R}^{11} \boldsymbol{\tau}(L) \mathbf{R}_{L}^{22} \boldsymbol{\tau}\left(L_{L}\right)\right]^{-1} & \\
& \left\{\mathbf{p}_{2 R}^{-}+\boldsymbol{\tau}\left(L_{R}\right)\left[\mathbf{R}_{R}^{11} \mathbf{p}_{2 R}^{+}+\mathbf{R}_{R}^{11} \boldsymbol{\tau}\left(L_{R}\right)\left(\mathbf{p}_{2 L}^{+}+\boldsymbol{\tau}\left(L_{L}\right) \mathbf{R}_{L}^{22} \mathbf{p}_{L}^{-}\right)\right]\right\} \\
\mathbf{a}_{2 R}^{+}= & \mathbf{p}_{2 L}^{+}+\boldsymbol{\tau}\left(L_{L}\right) \mathbf{R}_{L}^{22} \mathbf{p}_{2 L}^{-}+\boldsymbol{\tau}\left(L_{L}\right) \mathbf{R}_{L}^{22} \boldsymbol{\tau}\left(L_{L}\right) \mathbf{a}_{2 L}^{-}
\end{aligned}
$$


Therefore, the total charge is

$$
Q=\tilde{\mathbf{K}}^{\varphi \varphi} v L-\int_{0}^{L} \tilde{\mathbf{K}}_{0}^{\varphi u} \mathbf{q}(z)-\jmath k \tilde{\mathbf{K}}_{1}^{\varphi u} \mathbf{q}(z) \mathrm{d} z
$$

where

$$
\mathbf{q}\left(z_{i}\right)=\boldsymbol{\Phi}^{+} \mathbf{a}_{2 R}^{+}+\boldsymbol{\Phi}^{-} \mathbf{a}_{2 L}^{-}
$$

The integral in Eq. (55) can be evaluated numerically.

\section{Convergence}

Since the cross-section is discretised, the frequency range in which the results are credible is related to the density of the mesh. To determine how many elements are needed in a desired frequency range, one can check the convergence of the cut-off frequencies of the higher-order wave modes. This can be conveniently performed using the SAFE governing equation from Eq. (24) by setting $k=0$. The cut-off frequencies can then be found from

$$
\left(\mathbf{K}_{0}-\omega^{2} \mathbf{M}\right) \overline{\mathbf{q}}=\mathbf{0}
$$

The element size $L_{\max }$ ensuring that the cut-off frequencies converge is related to the bulk shear wavelength $\lambda_{T}[53,54]$

$$
L_{\max }=\lambda_{T} / \gamma
$$

where $\gamma=4$ for quadratic elements, and $\gamma=10$ for linear elements.

For a free rectangular piezoelectric actuator $(0.03 \times 0.003 \times 0.1 \mathrm{~m})$, made of Noliac NCE40 material (see Table 2 for material properties) and meshed using 8-node biquadratic quadrilaterals, Eq. (58) indicates that a structured mesh grid of $18 \times 2$ elements is sufficient. Below we verify that result by calculating the response to a driving voltage for increasing mesh densities in the frequency range $1-300 \mathrm{kHz}$.

The results for the end-displacement at the middle of the cross-section and the electrical admittance of the actuator as a response to a driving voltage are presented in Fig. 7. Both mechanical and electrical responses are shown to converge with increasing mesh density, proving the element formulation valid. 
(a)
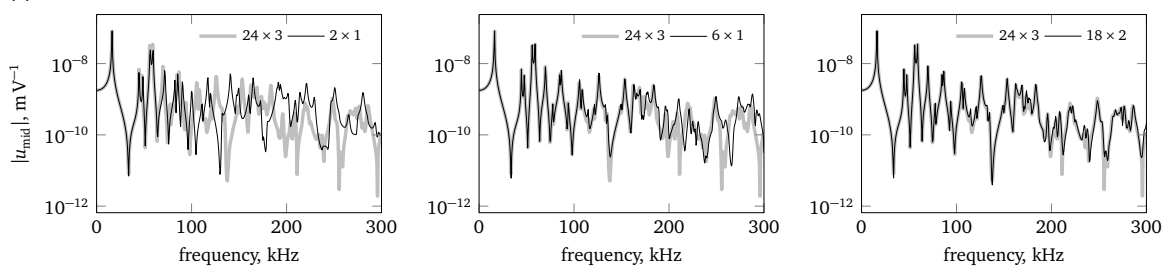

(b)
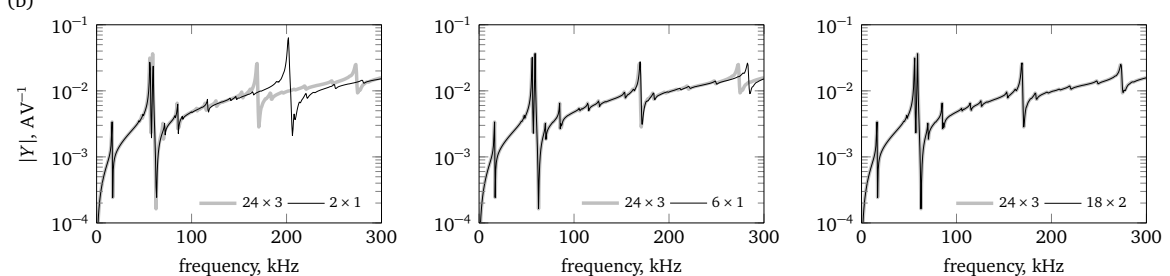

Figure 7: Response for different mesh configurations denoted in the legend as elements along $x \times$ elements along $y$. In each graph the results for a particular mesh are compared with a benchmark case in light grey: (a) displacement; (b) electrical admittance.

\section{Numerical verification}

Before the experimental validation is presented, the developed methodology is verified against numerical results from a commercial FE package (ABAQUS) and against a model based on the spectral element formulation from the open literature $([6])$. Note that one of the considered cases involves a more complex waveguide configuration than the illustrative example presented in Sec. 5.2. Derivation of the equations proceeds, however, along the same lines. For simplicity, a generalised piecewise formulation can be used as given in [51].

\subsection{Verification with FE}

Structural configurations for the benchmark cases are depicted in Fig. 8 and the corresponding material properties are listed in Table 1 and Table 2. The cross-sections in both the FE and SAFE are meshed using structured mesh (configurations in Fig. 8) and the element size along the propagation direction in FE is chosen to be $0.5 \mathrm{~mm}$ providing at least 4 quadratic elements per the shortest wavelength. The results of the comparison are shown in Fig. 9 and Fig. 10 
(a)
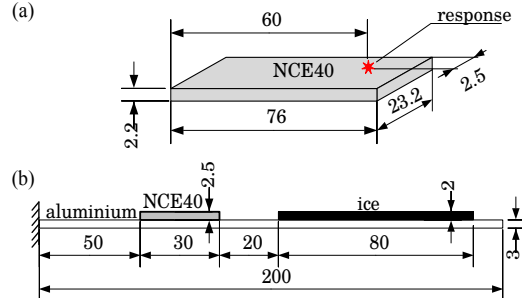

Cross-section mesh configurations:

SAFE: $8 \times 2$ (8-node biquadratic quadrilateral)

ABAQUS: 8x2 (C3D20E)

Cross-section mesh configurations: SAFE: 6 elements through each layer (3-node quadratic) ABAQUS: 6 elements through each layer (CPE8 and CPE8E)

Figure 8: Structural configurations for the numerical verification: (a) case 1; (b) case 2.

Table 1: Isotropic material constants used in the paper.

\begin{tabular}{lllll}
\hline Material & $E, \mathrm{GPa}$ & $\nu$ & $\rho, \mathrm{kg} \mathrm{m}^{-3}$ & $\eta, \%$ \\
\hline Aluminium & 69 & 0.33 & 2700 & 0.5 \\
Ice & 8.3 & 0.351 & 900 & 1 \\
Steel & 163 & 0.3 & 8000 & 0.01 \\
Epoxy & 15 & 0.4 & 1000 & 0.01 \\
\hline
\end{tabular}

showing very good agreement between the wave-based technique presented in this paper and conventional FE simulations.

\subsection{Verification against a beam model from the open literature}

In this section we compare the presented methodology with a model from the open literature [6] based on the spectral element formulation. In [6] the authors study a $0.6 \mathrm{~m}$ long and $2 \mathrm{~mm}$ thick aluminium cantilever beam excited by a piezoelectric actuator (PZT-5A, $0.2 \mathrm{~m}$ long and $0.5 \mathrm{~mm}$ thick, mounted $0.2 \mathrm{~m}$ from the fixed end). Their formulation employs the Timoshenko and Mindlin-Hermann theories with the piezoelectric excitation being represented by a pair of equivalent forces.

The response to the piezoelectric excitation computed using the model based on [6] is compared to the SAFE model presented in this paper and to a full 3D finite element model (as a benchmark) in Fig. 11. The longitudinal responses in Fig. 11(a) agree very well except for two regions: around 20 and $27 \mathrm{kHz}$. For the out-of-plane response in Fig. 11(b), all presented methods provide the 
Table 2: Piezoelectric material constants used in the paper.

\begin{tabular}{lll}
\hline Property & $\mathrm{NCE} 40$ & $\mathrm{PZT}-5 \mathrm{~A}$ \\
\hline$\rho, \mathrm{kg} \mathrm{m}^{-3}$ & 7850 & 7750 \\
$\eta, \%$ & 0.7 & 0 \\
$c_{x x x x}^{E}=c_{z z z z}^{E}, \mathrm{GPa}$ & 126.35 & 121 \\
$c_{x x y y}^{E}=c_{y y z z}^{E}, \mathrm{GPa}$ & 58.68 & 75.2 \\
$c_{y y y y}^{E}, \mathrm{GPa}^{E}$ & 99.88 & 111 \\
$c_{x x z z}^{E}, \mathrm{GPa}^{E}$ & 62.93 & 75.4 \\
$c_{x z x z}^{E}, \mathrm{GPa}^{E}$ & 31.71 & 21.1 \\
$c_{y z y z}^{E}=c_{x y x y}^{E}, \mathrm{GPa}^{E}$ & $\mathrm{n} 36.77$ & 22.6 \\
$\varepsilon_{x x}^{\epsilon}, \mathrm{F} \mathrm{m}^{-1} \times 10^{-9}$ & 5.5 & 8.119 \\
$\varepsilon_{z z}^{\epsilon}, \mathrm{F} \mathrm{m}^{-1} \times 10^{-9}$ & 5.5 & 8.119 \\
$\varepsilon_{y y}^{\epsilon}, \mathrm{F} \mathrm{m}^{-1} \times 10^{-9}$ & 5.196 & 7.349 \\
$e_{y, x x x x}, \mathrm{~N} \mathrm{~V}^{-1} \mathrm{~m}^{-1}$ & -3.239 & -5.46 \\
$e_{y, z z z z}, \mathrm{~N} \mathrm{~V}^{-1} \mathrm{~m}^{-1}$ & -3.239 & -5.46 \\
$e_{z, y z y z}, \mathrm{~N} \mathrm{~V}^{-1} \mathrm{~m}^{-1}$ & 13.075 & 13.198 \\
$e_{x, x y x y}, \mathrm{~N} \mathrm{~V}^{-1} \mathrm{~m}^{-1}$ & 13.075 & 13.198 \\
$e_{y, y y y y}, \mathrm{~N} \mathrm{~V}^{-1} \mathrm{~m}^{-1}$ & 16.335 & 15.796 \\
\hline
\end{tabular}

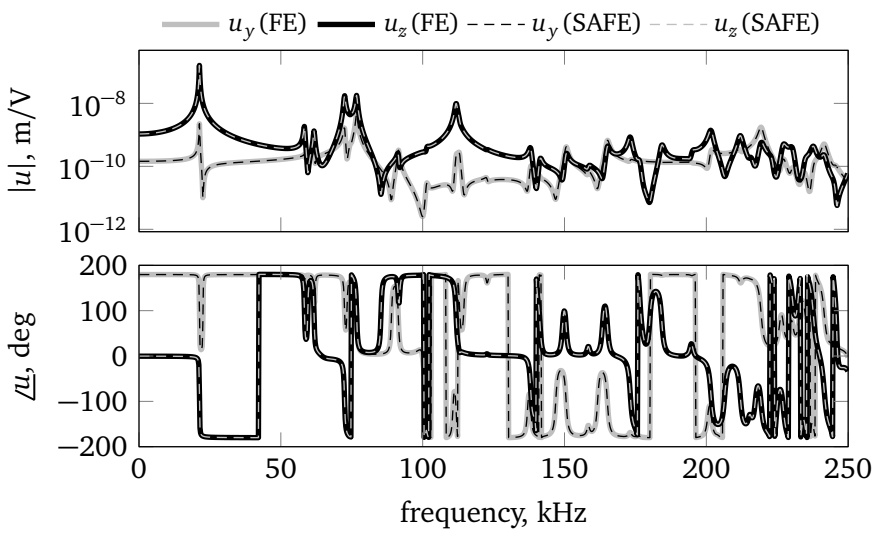

Figure 9: Verification case 1: velocity at a point on the electrode surface of a free PZT actuator in response to a driving voltage. 

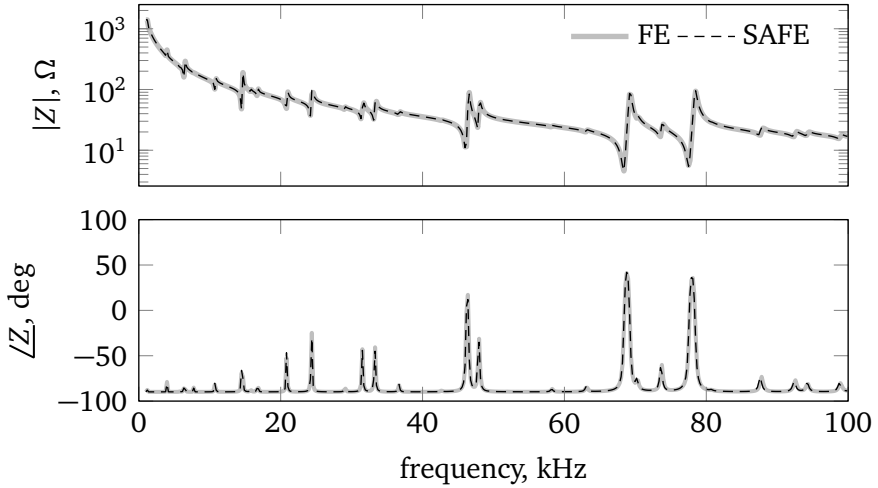

Figure 10: Verification case 2: electrical impedance of a PZT actuator bonded to an aluminium cantilever with an ice patch.
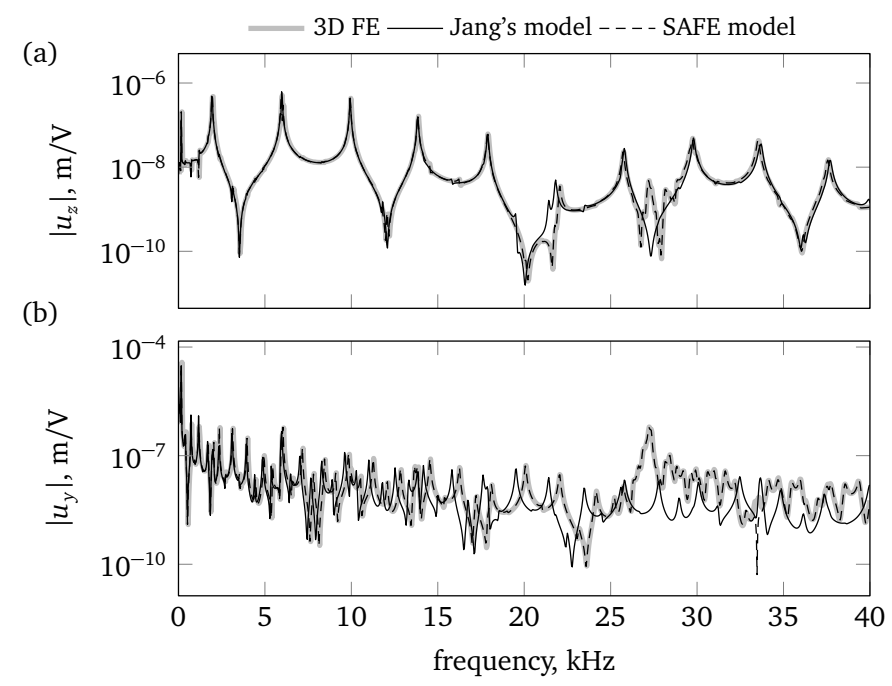

Figure 11: Verification against a model based on [6] and 3D FE (as a benchmark) - response to a driving voltage: a) longitudinal displacement; b) transverse displacement. 
(a)

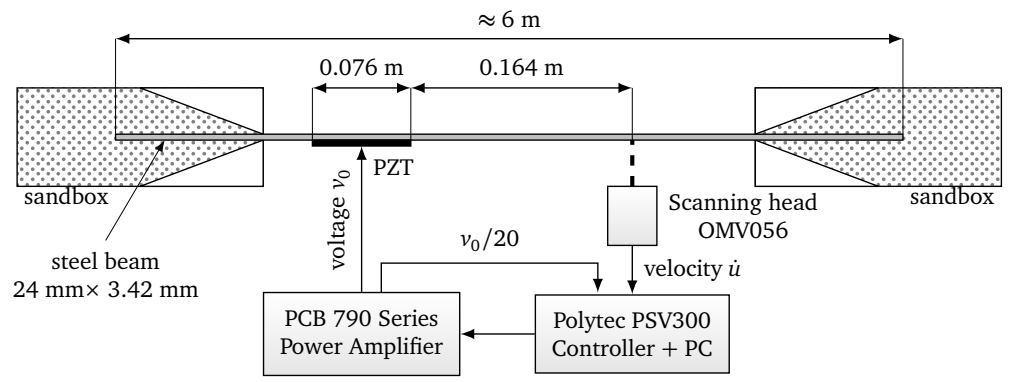

(b)

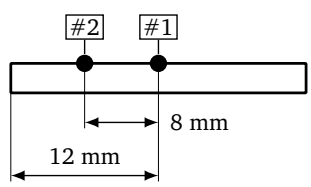

Figure 12: Experimental setup for the validation of the model: (a) schematic diagram; (b) response locations on the cross-section plane.

same results up to $5 \mathrm{kHz}$. Further in frequency, the discrepancy between the models increases and around $10 \mathrm{kHz}$ starts to be significant. At around $27 \mathrm{kHz}$ higher-order waves start to propagate in the structure and significantly alter its response. These effects are well captured by the SAFE model and 3D FE only. Whilst the methodology from [6] is fast and reliable for a range of applications at low frequencies, the SAFE model allows for accurate calculations at mid- and high-frequencies with the ability to represent the dynamics of piezo-equipped structures correctly.

\section{Experimental validation}

The framework proposed in this paper was validated with an experiment on a piezo-equipped beam with emulated anechoic terminations. A schematic diagram of the experimental set-up is shown in Fig. 12, whereas corresponding material properties are listed in Table 1 and Table 2. The piezoelectric actuator $(0.076 \mathrm{~m} \times 0.024 \mathrm{~m} \times 0.0022 \mathrm{~m})$ was made of Noliac NCE40 material and attached to the beam using a thin (ca. $0.1 \mathrm{~mm}$ ) layer of silvered epoxy (Circuit Works CW2400). The properties of NCE40 from [55] were updated based on the free-free actuator impedance measurement to provide the closest match (within stated tolerances) giving the values listed in Table 2. The properties of steel resulted from model updating based on an impact hammer test. 

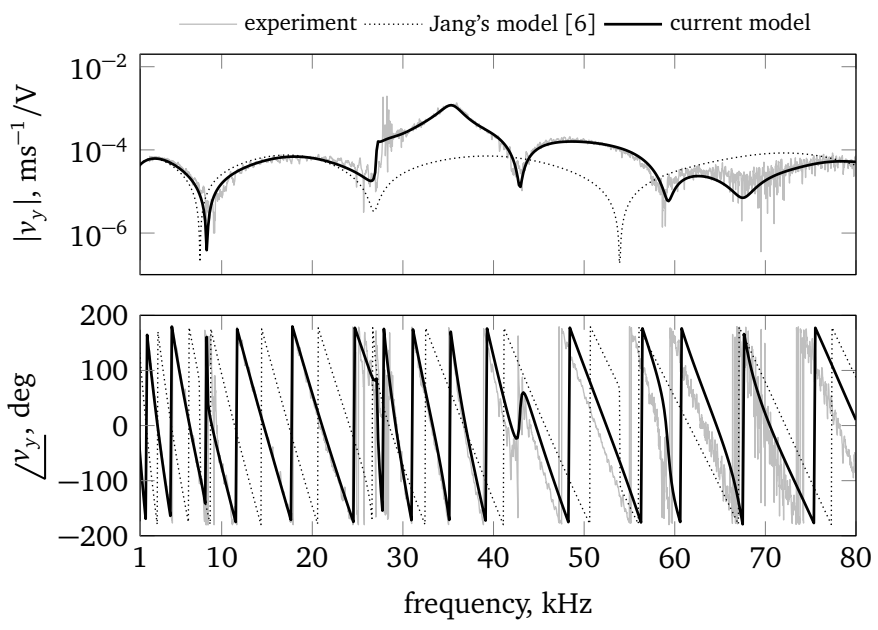

Figure 13: Magnitude and phase of the out-of-plane velocity with respect to the driving voltage measured at scan point \#1 (see Fig. 12).

The response was measured at a few locations across the width of the beam using a Polytec PSV300 laser vibrometer, two of which are presented below (see Fig. 12). The ends of the beam were placed in boxes filled with sand in such a way that the thickness of the sand cover was gradually increased to ensure a smooth change in the mechanical impedance of the boundary and minimise any reflections.

A corresponding wave model consisted of 3 wave elements and was exactly the same as described in Sec. 5.2. Each cross-section was modelled with six 8-node biquadratic quadrilateral elements across the width and one element through the thickness of each layer.

Illustrative recorded responses from scan point \#1 and \#2 are compared with the wave model predictions in Fig. 13 and Fig. 14, respectively. The choice of scan points demonstrates the variation of the response across the width of the beam especially between 30 and $40 \mathrm{kHz}$.

The response at mid-width of the beam (point \#1) is shown in Fig. 13. At low frequencies it follows the behaviour expected from the approximate theories with simple bending being dominant. The sudden jump in magnitude at around 

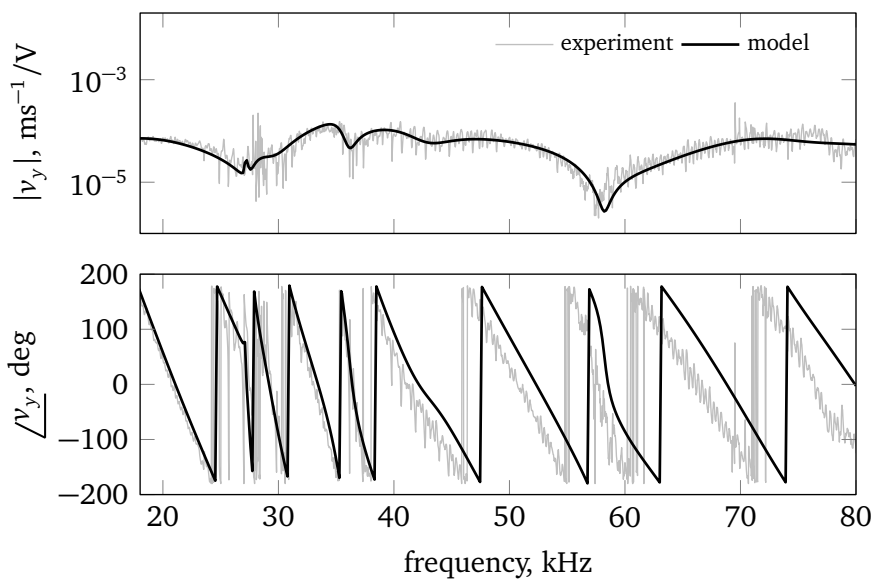

Figure 14: Magnitude and phase of the out-of-plane velocity with respect to the driving voltage measured at scan point \#2 (see Fig. 12).

$27 \mathrm{kHz}$ corresponds to the cut-off frequency of the first across-width bending wave in the steel beam (denoted as U-wave). At around $35 \mathrm{kHz}$ the response reaches its maximum which is related to the cut-off frequency of the U-wave in the PZT-covered section. The PZT-covered section is then at resonance. Predictions obtained with the model based on [6] (see Sec. 7.2) included in Fig. 13 agree with the experiment up to ca. $5 \mathrm{kHz}$ but deviate at higher frequencies as the dynamics of the PZT-covered section cannot be captured by simple beam theories.

Fig. 14 shows the response measured at a location close to the node of the Uwave (point \#2). Consequently, there is no significant increase in the amplitude between 27 and $35 \mathrm{kHz}$. Our model is shown to represent the experimental response very well including higher-order wave effects.

The wave absorption in the sandboxes was observed to have a nonlinear character and depended significantly on the amplitude of the waves. Large amplitude waves near the cut-off frequency are poorly absorbed by the sandboxes. Nevertheless, despite the absorption being imperfect, the mobilities presented in Fig. 13 and Fig. 14 capture predominantly the effect of the incident waves and show a very good agreement between the modelling approach presented in 
(a)

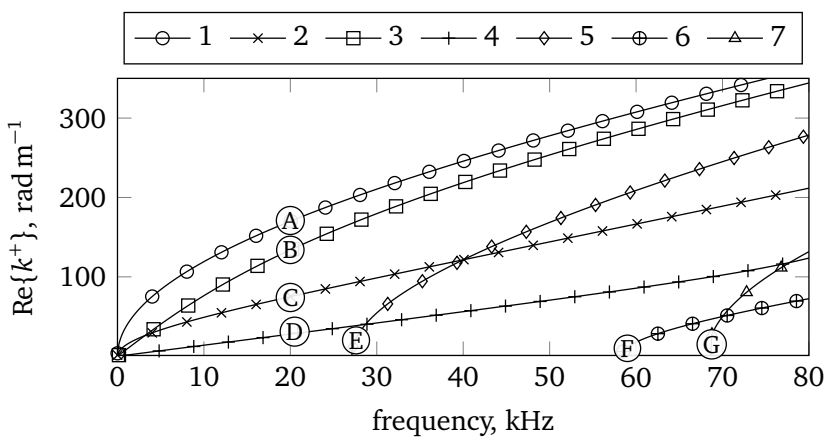

(b)

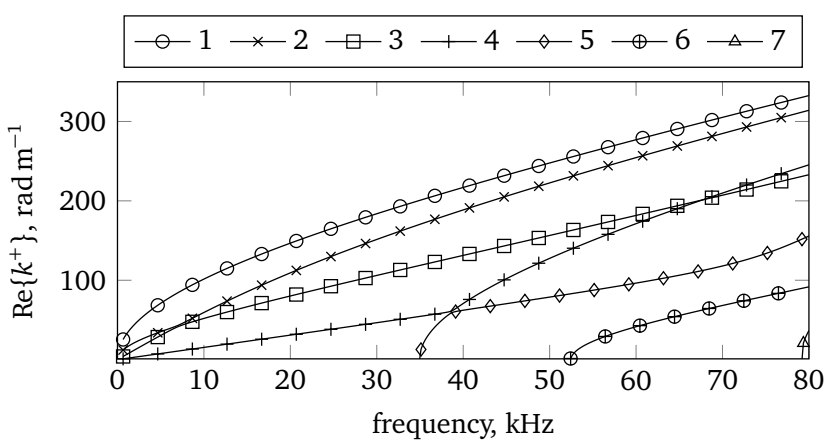

Figure 15: Dispersion curves for the cross-sections used in the validation experiment: (a) steel beam; (b) PZT-epoxy-steel beam.

this paper and the experimental data.

\section{Wave analysis of the experimental case}

In this section the experimental case is analysed from a wave perspective using the SAFE model. The wave-based nature of the proposed model renders insight into the wave characteristics of the structure and the wave composition of the FRF. The dispersion curves for the cross-sections used in the experiment are presented in Fig. 15. At each frequency the wavenumbers are arranged according to the WAC matrix (Wave Assurance Criterion [47]) ensuring the continuity of the wave mode shape along the dispersion curve.

Points A-G as marked in Fig. 15 are used to describe the displacement wave mode shape associated with particular waves in a steel beam which are shown in 
A
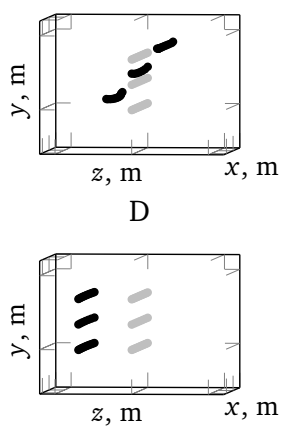

B
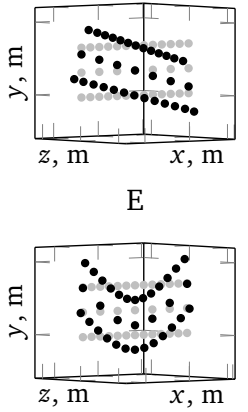

$\mathrm{G}$

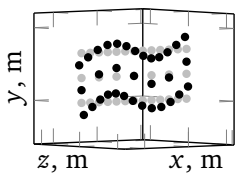

C
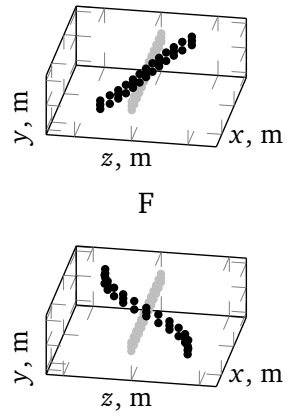

Figure 16: Predicted wave displacement mode shapes for the steel beam used in the experiment associated with the points highlighted in Fig. 15. The geometry scale is not preserved in order to facilitate visualisation.

Fig. 16. When the PZT layer is present the cut-off frequencies of the transverse across-width bending waves are shifted up since the bending stiffness of the cross-section is increased.

The wave model enables decomposition of the response into contributions from particular waves. The piezoelectric actuator in the chosen configuration is able to activate only three of the seven waves in the frequency range of interest (Fig. 15). The velocities associated with each propagating wave (as magnitudes) are shown in Fig. 17 with the legend and labelling convention adopted from Fig. 15. The FRF corresponds to the surface out-of-plane velocity at scan point \#4. One can observe that at low frequencies the fundamental flexural wave is prevalent in the response. When the U-wave cuts off at $27 \mathrm{kHz}$ it locally dominates the FRF and from that frequency onwards it is mainly the two aforementioned waves that contribute to the overall response. The influence of the compressional wave is very small as expected from the direction of the velocity measurement (out-of-plane). 


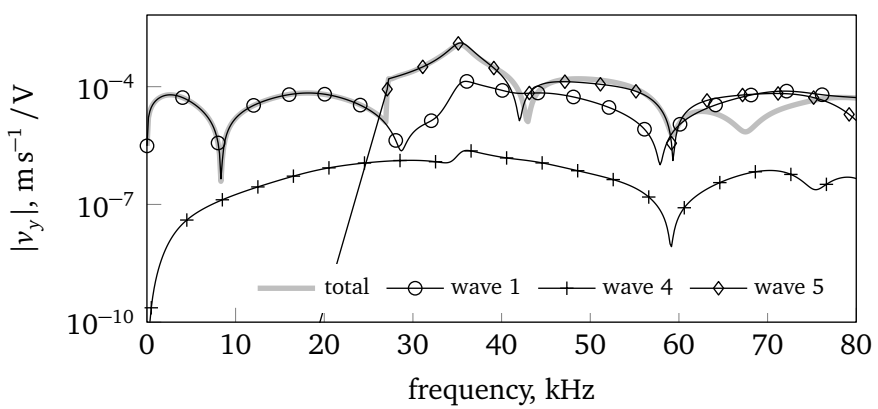

Figure 17: Wave composition of the response measured at scan point \#1 (Fig. 12) - shown as magnitudes of modal contributions.

\section{Conclusions}

In this paper, a new wave-based methodology for modelling piezoelectric excitation in waveguides was proposed resulting from the coupling of the semianalytical finite element method and the analytical wave approach. We developed a piezoelectric semi-analytical finite element suited to representing electroded piezoelectric actuators and outlined the mathematical framework for modelling distributed voltage excitation. The actuator-covered element was coupled to the other sections of the waveguide using scattering matrices. A few numerical examples were used to verify the model against conventional finite element simulations and a model from the open literature, which highlighted the physical insight gained with the proposed method. Finally, we conducted an experimental validation which showed very good agreement between the predictions of the model and the measured responses of a piezo-actuated beam with emulated anechoic terminations.

\section{Acknowledgements}

The authors thank Prof. Svante Finnveden and Dr. Guido Zarini for inspiring discussions on the SAFE method. This research has been possible thanks to the University of Southampton Postgraduate Research Studentship Programme. Financial support of Structural Funds in the Operational Programme 
- Innovative Economy (IE OP) financed from the European Regional Development Fund - Project "Modern material technologies in aerospace industry", Nr POIG.01.01.02-00-015/08-00 is gratefully acknowledged. The authors also acknowledge the use of the IRIDIS High Performance Computing Facility at the University of Southampton. The data supporting this study are openly available from the University of Southampton repository at http://dx.doi.org/10.5258/SOTON/392801.

\section{Appendix A. Static condensation of the internal potentials}

A static condensation scheme is briefly recalled here following [40, 41, 43] and extended to the case of wavenumber-dependent matrices. For the sake of brevity we write the SAFE matrices in the following manner:

$$
\begin{aligned}
\mathbf{K}^{u u}(k) & =\mathbf{K}_{0}-\jmath k \mathbf{K}_{1}-(-\jmath k)^{2} \mathbf{K}_{2} \\
\mathbf{K}^{u \varphi}(k) & =\mathbf{K}_{0}^{u \varphi}+\jmath k \mathbf{K}_{1}^{u \varphi} \\
\mathbf{K}^{\varphi u}(k) & =\mathbf{K}_{0}^{\varphi u}-\jmath k \mathbf{K}_{1}^{\varphi u}
\end{aligned}
$$

In the following the index $i$ denotes the internal nodes, index $v$ denotes the nodes on the powered electrode and finally the index 0 denotes the nodes on the grounded electrode. Eq. (20) is then rewritten as follows:

$$
\left[\begin{array}{cccc}
\mathbf{K}^{u u}(k)-\omega^{2} \mathbf{M} & \mathbf{K}_{v}^{u \varphi}(k) & \mathbf{K}_{0}^{u \varphi}(k) & \mathbf{K}_{i}^{u \varphi}(k) \\
\mathbf{K}_{v}^{\varphi u}(k) & -\mathbf{K}_{v v}^{\varphi \varphi} & -\mathbf{K}_{v 0}^{\varphi \varphi} & -\mathbf{K}_{v i}^{\varphi \varphi} \\
\mathbf{K}_{0}^{\varphi u}(k) & -\mathbf{K}_{0 v}^{\varphi \varphi} & -\mathbf{K}_{00}^{\varphi \varphi} & -\mathbf{K}_{0 \mathrm{i}}^{\varphi \varphi} \\
\mathbf{K}_{i}^{\varphi u}(k) & -\mathbf{K}_{i V}^{\varphi \varphi} & -\mathbf{K}_{\mathrm{i} 0}^{\varphi \varphi} & -\mathbf{K}_{i i}^{\varphi \varphi}
\end{array}\right]\left\{\begin{array}{c}
\overline{\mathbf{q}} \\
\overline{\boldsymbol{\varphi}}_{v} \\
\overline{\boldsymbol{\varphi}}_{0} \\
\overline{\boldsymbol{\varphi}}_{i}
\end{array}\right\}=\left\{\begin{array}{c}
\overline{\mathbf{p}} \\
-\overline{\mathbf{\Upsilon}}_{v} \\
\mathbf{0} \\
\mathbf{0}
\end{array}\right\}
$$

We assume that there are no external forces and remove rows and columns associated with the grounded electrode $\overline{\varphi_{0}}$ since $\overline{\varphi_{0}}=0$. Potentials at the internal nodes can be extracted from the last equation:

$$
\overline{\boldsymbol{\varphi}}_{i}=\left(\mathbf{K}_{i i}^{\varphi \varphi}\right)^{-1}\left(\mathbf{K}_{i}^{\varphi u}(k) \overline{\mathbf{q}}-\mathbf{K}_{i V}^{\varphi \varphi} \overline{\boldsymbol{\varphi}}_{v}\right)
$$

Eq. (A.3) is substituted into Eq. (A.2) and the matrices are expanded according to the shorthand notation introduced in Eq. (A.1). The resulting equations are rearranged and grouped with respect to the order of the wavenumber. 
Consequently, one can write the expressions for condensed matrices

$$
\begin{aligned}
\tilde{\mathbf{K}}_{0} & =\mathbf{K}_{0}+\mathbf{K}_{0 i}^{u \varphi}\left(\mathbf{K}_{i i}^{\varphi \varphi}\right)^{-1} \mathbf{K}_{0 \mathrm{i}}^{\varphi u} \\
\tilde{\mathbf{K}}_{1} & =\mathbf{K}_{1}+\left[\mathbf{K}_{0 i}^{u \varphi}\left(\mathbf{K}_{i i}^{\varphi \varphi}\right)^{-1} \mathbf{K}_{1 i}^{\varphi u}-\mathbf{K}_{1 i}^{u \varphi}\left(\mathbf{K}_{i i}^{\varphi \varphi}\right)^{-1} \mathbf{K}_{0 i}^{\varphi u}\right] \\
\tilde{\mathbf{K}}_{2} & =\mathbf{K}_{2}+\mathbf{K}_{1 i}^{u \varphi}\left(\mathbf{K}_{i i}^{\varphi \varphi}\right)^{-1} \mathbf{K}_{1 i}^{\varphi u} \\
\tilde{\mathbf{K}}_{0}^{u \varphi} & =\tilde{\mathbf{K}}_{0}^{\varphi u^{\top}}=\left[\mathbf{K}_{0 v}^{u \varphi}-\mathbf{K}_{0 i}^{u \varphi}\left(\tilde{\mathbf{K}}_{i i}^{\varphi \varphi}\right)^{-1} \mathbf{K}_{i v}^{\varphi \varphi}\right] \mathbf{I}_{\mathbf{v}} \\
\tilde{\mathbf{K}}_{1}^{u \varphi} & =\tilde{\mathbf{K}}_{1}^{\varphi u^{\top}}=\left[\mathbf{K}_{1 v}^{u \varphi}-\mathbf{K}_{1 i}^{u \varphi}\left(\mathbf{K}_{i i}^{\varphi \varphi}\right)^{-1} \mathbf{K}_{i V}^{\varphi \varphi}\right] \mathbf{I}_{\mathbf{v}} \\
\tilde{\mathbf{K}}^{\varphi \varphi} & =\mathbf{I}_{\mathbf{v}}^{\top}\left[\mathbf{K}_{v v}^{\varphi \varphi}-\mathbf{K}_{V i}^{\varphi \varphi}\left(\mathbf{K}_{i i}^{\varphi \varphi}\right)^{-1} \mathbf{K}_{i V}^{\varphi \varphi}\right] \mathbf{I}_{\mathbf{v}} \\
\tilde{\mathbf{K}}_{f} & =\mathbf{K}_{f}+\mathbf{K}_{1 i}^{u \varphi}\left(\mathbf{K}_{i i}^{\varphi \varphi}\right)^{-1} \mathbf{K}_{0 i}^{\varphi u}
\end{aligned}
$$

In the above equation the contributions of all nodes on the powered electrode are summed up since voltage is constant over the driving electrode. To accomplish that appropriate matrices are multiplied by vector $\mathbf{I}_{\mathbf{v}}=\left[\begin{array}{llll}1 & 1 & \ldots & 1\end{array}\right]^{\top}$ of the length equal to the number of nodes on the powered piezoelectric electrode [41].

\section{References}

[1] G. Huang, F. Song, X. Wang, Quantitative Modeling of Coupled PiezoElastodynamic Behavior of Piezoelectric Actuators Bonded to an Elastic Medium for Structural Health Monitoring: A Review, Sensors 10 (2010) $3681-3702$.

[2] J. Moll, M. V. Golub, E. Glushkov, N. Glushkova, C.-P. Fritzen, Nonaxisymmetric Lamb wave excitation by piezoelectric wafer active sensors, Sensor Actuat A-Phys 174 (2012) 173-180.

[3] E. F. Crawley, J. De Luis, Use of piezoelectric actuators as elements of intelligent structures, AIAA J 25 (1987) 1373-1385.

[4] U. Lee, J. Kim, Dynamics of elastic-piezoelectric two-layer beams using spectral element method, Int J Solids Sturct 37 (2000) 4403-4417. 
[5] H. W. Park, E. J. Kim, K. L. Lim, H. Sohn, Spectral element formulation for dynamic analysis of a coupled piezoelectric wafer and beam system, Comput Struct 88 (2010) 567-580.

[6] I. Jang, I. Park, U. Lee, Spectral element modeling and analysis of the dynamics and guided waves in a smart beam with a surface-bonded PZT layer, J Mech Sci Technol 28 (2014) 1229-1239.

[7] I. Park, U. Lee, Dynamic analysis of smart composite beams by using the frequency-domain spectral element method, J Mech Sci Technol 26 (2012) 2511-2521. Park2012.

[8] V. Giurgiutiu, Structural health monitoring: with piezoelectric wafer active sensors, Access Online via Elsevier, 2007.

[9] X. Lin, F. G. Yuan, Diagnostic Lamb waves in an integrated piezoelectric sensor/actuator plate: analytical and experimental studies, Smart Mater Struct 10 (2001) 907.

[10] E. Moulin, J. Assaad, C. Delebarre, D. Osmont, Modeling of Lamb waves generated by integrated transducers in composite plates using a coupled finite elementnormal modes expansion method, J Acoust Soc Am 107 (2000) $87-94$.

[11] A. Raghavan, C. E. S. Cesnik, Finite-dimensional piezoelectric transducer modeling for guided wave based structural health monitoring, Smart Mater Struct 14 (2005) 1448-1461.

[12] V. Giurgiutiu, Tuned Lamb Wave Excitation and Detection with Piezoelectric Wafer Active Sensors for Structural Health Monitoring, J Intel Mat Syst Str 16 (2005) 291-305.

[13] F. L. d. Scalea, S. Salamone, Temperature effects in ultrasonic Lamb wave structural health monitoring systems, J Acoust Soc Am 124 (2008) 161174. 
[14] G. Santoni-Bottai, V. Giurgiutiu, Exact Shear-Lag Solution for Guided Waves Tuning with Piezoelectric-Wafer Active Sensors, AIAA Journal 50 (2012) 2285-2294.

[15] X. Wang, S. Meguid, On the electroelastic behaviour of a thin piezoelectric actuator attached to an infinite host structure, Int J Solids Sturct 37 (2000) $3231-3251$.

[16] X. D. Wang, G. L. Huang, Wave propagation generated by piezoelectric actuators attached to elastic substrates, Acta Mech 183 (2006) 155-176.

[17] E. Glushkov, N. Glushkova, O. Kvasha, W. Seemann, Integral equation based modeling of the interaction between piezoelectric patch actuators and an elastic substrate, Smart Mater Struct 16 (2007) 650-664.

[18] Y. Kim, S. Ha, F.-K. Chang, Time-Domain Spectral Element Method for Built-In Piezoelectric-Actuator-Induced Lamb Wave Propagation Analysis, AIAA J 46 (2008) 591-600.

[19] S. Ha, F.-K. Chang, Optimizing a spectral element for modeling PZTinduced Lamb wave propagation in thin plates, Smart Mater Struct 19 (2010) 015015 .

[20] W. Ostachowicz, P. Kudela, M. Krawczuk, A. Zak, Guided Waves in Structures for SHM: The Time-Domain Spectral Element Method, John Wiley \& Sons, Ltd, 2012.

[21] P. Paćko, T. Bielak, A. B. Spencer, W. J. Staszewski, T. Uhl, K. Worden, Lamb wave propagation modelling and simulation using parallel processing architecture and graphical cards, Smart Mater Struct 21 (2012) 075001.

[22] L. Cremer, M. Heckl, Structure-borne sound; structural vibrations and sound radiation at audio frequencies, Springer-Verlag, 1973.

[23] B. R. Mace, Wave reflection and transmission in beams, J Sound Vib 97 (1984) 237-246. 
[24] C. Li, C. Song, H. Man, E. T. Ooi, W. Gao, 2d dynamic analysis of cracks and interface cracks in piezoelectric composites using the SBFEM, Int J Solids Sturct 51 (2014) 2096-2108.

[25] H. Gravenkamp, C. Birk, C. Song, Simulation of elastic guided waves interacting with defects in arbitrarily long structures using the Scaled Boundary Finite Element Method, J Comput Phys 295 (2015) 438-455.

[26] G. R. Liu, J. D. Achenbach, A Strip Element Method for Stress Analysis of Anisotropic Linearly Elastic Solids, J Appl Mech 61 (1994) 270-277.

[27] G. R. Liu, Z. C. Xi, K. Y. Lam, H. M. Shang, A Strip Element Method for Analyzing Wave Scattering by a Crack in an Immersed Composite Laminate, J Appl Mech 66 (1999) 898-903.

[28] M. Nenning, Infinite Elements for Elasto- and Poroelastodynamics, volume 8 of Monographic Series TU GRAZ - Computation in Engineering and Science, Verlag der Technischen Universitt Graz, 2010.

[29] P. E. Lagasse, Higher-order finite-element analysis of topographic guides supporting elastic surface waves, J Acoust Soc Am 53 (1973) 1116-1122.

[30] B. Aalami, Waves in Prismatic Guides of Arbitrary Cross Section, J Appl Mech 40 (1973) 1067-1072.

[31] T. Hayashi, W.-J. Song, J. L. Rose, Guided wave dispersion curves for a bar with an arbitrary cross-section, a rod and rail example, Ultrasonics 41 (2003) 175-183.

[32] V. Damljanović, R. L. Weaver, Forced response of a cylindrical waveguide with simulation of the wavenumber extraction problem, J Acoust Soc Am 115 (2004) 1582.

[33] I. Bartoli, A. Marzani, F. Lanza di Scalea, E. Viola, Modeling wave propagation in damped waveguides of arbitrary cross-section, J Sound Vib 295 (2006) 685-707. 
[34] H. Gao, Ultrasonic guided wave mechanics for composite material structural health monitoring, Ph.D. thesis, Pennsylvania State University, 2007.

[35] B. Le Crom, M. Castaings, Shear horizontal guided wave modes to infer the shear stiffness of adhesive bond layers, J Acoust Soc Am 127 (2010) 2220.

[36] B. Auld, Acoustic Fields in Elastic Solids, Vol. I, II, Wiley-Interscience, 1973.

[37] M. Petyt, Introduction to Finite Element Vibration Analysis, 2nd edition ed., Cambridge University Press, New York, 2010.

[38] P. Gaudenzi, Smart Structures: Physical Behaviour, Mathematical Modelling and Applications, John Wiley \& Sons, 2009.

[39] P. Gaudenzi, K.-J. Bathe, An Iterative Finite Element Procedure for the Analysis of Piezoelectric Continua, J Intel Mat Syst Str 6 (1995) 266-273.

[40] H. Allik, T. J. R. Hughes, Finite element method for piezoelectric vibration, Int J Numer Meth Eng 2 (1970) 151-157.

[41] J. Kocbach, Finite Element Modeling of Ultrasonic Piezoeelctric Transducers, Ph.D. thesis, University of Bergen, 2000.

[42] V. Piefort, Finite element modelling of piezoelectric active structures, Ph.D. thesis, Universit Libre de Bruxelles, 2001.

[43] D. Boucher, M. Lagier, C. Maerfeld, Computation of the Vibrational Modes for Piezoelectric Array Transducers Using a Mixed Finite ElementPerturbation Method, IEEE T Son Ultrason 28 (1981) 318-329.

[44] F. Tisseur, K. Meerbergen, The Quadratic Eigenvalue Problem, SIAM Rev 43 (2001) 235-286.

[45] B. R. Mace, D. Duhamel, M. J. Brennan, L. Hinke, Finite element prediction of wave motion in structural waveguides, The Journal of the Acoustical Society of America 117 (2005) 2835-2843. 
[46] F. Treyssde, Elastic waves in helical waveguides, Wave Motion 45 (2008) 457-470.

[47] Y. Waki, On the application of finite element analysis to wave motion in one-dimensional waveguides, Ph.D. thesis, University of Southampton, 2007.

[48] J. M. Renno, B. R. Mace, On the forced response of waveguides using the wave and finite element method, J Sound Vib 329 (2010) 5474-5488.

[49] P. M. Morse, Theoretical Acoustics, Princeton University Press, 1986.

[50] E. Skudrzyk, The Foundations of Acoustics - Basic Mathematics and Basic Acoustics, 1st ed., Springer-Verlag, Wien - New York, 1971.

[51] M. Kalkowski, Piezo-actuated structural waves for delaminating surface accretions, Ph.D. thesis, University of Southampton, 2015.

[52] N. Harland, B. Mace, R. Jones, Wave propagation, reflection and transmission in tunable fluid-filled beams, J Sound Vib 241 (2001) 735-754.

[53] J. M. Galán, R. Abascal, Numerical simulation of Lamb wave scattering in semi-infinite plates, Int J Numer Meth Eng 53 (2002) 1145-1173.

[54] A. Marzani, E. Viola, I. Bartoli, F. Lanza di Scalea, P. Rizzo, A semianalytical finite element formulation for modeling stress wave propagation in axisymmetric damped waveguides, J Sound Vib 318 (2008) 488-505.

[55] Noliac Group, 2014, Piezo materials datasheet, URL: http: //www.noliac.com/Files/Billeder/02\%20Standard/Ceramics/ Noliac_CEramics_NCE_datasheet.pdf. 